\title{
Sex differences in age- and episodic memory-related effects on task-based functional connectivity
}

Sivaniya Subramaniapillai ${ }^{1,2}$, Sricharana Rajagopal ${ }^{2}$, Elizabeth Ankudowich ${ }^{2,3}$, Stamatoula Pasvanis ${ }^{2}$, Bratislav Misic ${ }^{4}$, and M.Natasha Rajah ${ }^{1,2,5}$

${ }^{1}$ Department of Psychology, Faculty of Science, McGill University

${ }^{2}$ Brain Imaging Centre, Douglas Mental Health University Institute

${ }^{3}$ Integrated Program in Neuroscience, Faculty of Medicine, McGill University

${ }^{4}$ Montreal Neurological Institute, McGill University

${ }^{5}$ Department of Psychiatry, Faculty of Medicine, McGill University

Corresponding author:

M. Natasha Rajah

Room 2114 CIC Pavilion

Douglas Mental Health University Institute 6875 LaSalle Blvd

Montreal, QC, Canada H4H 1R3

maria.rajah@mcgill.ca 


\section{Abstract}

2 differences in the effect of normative aging on memory-related brain network dynamics. Here, we used

3 a data-driven multivariate partial least squares (PLS) connectivity analysis to identify similarities and

4 differences in the effect of biological sex on age- and memory-related differences in task-based fMRI

5 connectivity during encoding and retrieval of face-location associations (spatial context memory).

6 Aging was associated with episodic memory decline in both sexes, but there were no significant sex or

7 sex-by-age interactions in memory performance. The connectivity results show that men exhibited

8 greater between-network connectivity with advanced age, which was detrimental to memory

9 performance. Women exhibited reduced connectivity between visual and higher order cognitive

10 networks with advanced age, which was detrimental to memory performance. Therefore, there are sex

11 differences in the effect of age on episodic memory-related connectivity.

12 Key words: lifespan, sex differences, context memory, encoding, retrieval, fMRI, functional

13 connectivity 


\section{Introduction}

Our ability to encode, store, and retrieve information about past events in rich spatial and temporal contextual detail (episodic memory) is critical to our identity (Tulving, 2002). If we did not remember our past, we would have a poor understanding of ourselves and the environment, which would in turn impact our ability to plan for the future (Schacter et al., 2012), establish strong interpersonal relationships (Jing, Madore, \& Schacter, 2020; Mano et al., 2011), and participate in daily activities (Dong, Wilson, De Leon, \& Evans, 2010). Healthy aging is associated with episodic memory

decline (Grady \& Craik, 2000). These memory losses impair older adults' quality of life, daily

functioning, and sometimes lead to detrimental health effects (Mol et al., 2007; Mol, van Boxtel,

Willems, \& Jolles, 2006). Given that the proportion of elderly adults is increasing worldwide, there is

an urgent need to understand how normative aging influences memory and brain function. Such

knowledge provides the necessary groundwork for distinguishing normative from pathological

differences in memory and brain function and can provide insight for future intervention studies aimed at supporting episodic memory function in late life.

To this aim, there has been significant research directed at understanding how normative aging

affects episodic memory and associated brain function. Behavioural studies have shown that older

adults experience greater deficits in their ability to recollect spatial and temporal contextual details of

past events (i.e., context memory), compared to their ability to recognize previously encountered events

based functional magnetic resonance imaging (fMRI) activity have found that age-related differences in

episodic memory performance are associated with age differences in regional brain activation in a

distributed set of brain regions (e.g., medial temporal lobes [MTL], prefrontal cortex [PFC], inferior

parietal cortex [IPC]; Ankudowich, Pasvanis, \& Rajah, 2016, 2017; Grady et al., 2010; Hayama, 
cognition and behaviour depends on the dynamic interactions of large-scale neural networks (Friston,

1994; McIntosh, 2000; Mesulam, 1990; Sporns \& Betzel, 2016; Strother, Kanno, Rottenberg, Friston,

\& Ford, 1995), recent studies have increasingly focused on how age differences in inter-regional or inter-network correlations in brain activity (functional connectivity) relate to episodic memory ability.

time series between brain regions and canonical brain networks (Biswal, Zerrin Yetkin, Haughton, \&

how this relates to cognitive performance as assessed by neuropsychological tests or pre/post scan. In

general, these rsfMRI connectivity studies have found that older adults show greater inter-network and

reduced within-network functional connectivity compared to younger adults (Allen et al., 2011;

Antonenko \& Flöel, 2013; Betzel et al., 2014; Cao et al., 2014; Chan, Park, Savalia, Petersen, \& Wig,

2014; Damoiseaux, 2017; Ferreira et al., 2016; Geerligs, Renken, Saliasi, Maurits, \& Lorist, 2015;

Grady, Sarraf, Saverino, \& Campbell, 2016; King et al., 2018; Ng, Lo, Lim, Chee, \& Zhou, 2016;

Siman-Tov et al., 2017; Spreng, Stevens, Viviano, \& Schacter, 2016; Stumme, Jockwitz, Hoffstaedter,

Amunts, \& Caspers, 2020; Tian, Li, Wang, \& Yu, 2018). This loss of functional specialization with

aging is thought to reflect decreases in efficient information processing (Bullmore \& Sporns, 2012;

Sporns, 2013), and has been linked to reduced task performance on several cognitive domains,

including episodic memory (Chan et al., 2014; Damoiseaux et al., 2008; Geerligs et al., 2015; He et al.,

2012; Mevel et al., 2013; Onoda, Ishihara, \& Yamaguchi, 2012; Spreng et al., 2016).

Much of this research assessing functional connectivity differences with age have found an important relationship between the default mode network (DMN) and the dorsal attention network (DAN), particularly during cognitive task performance (Amer, Anderson, Campbell, Hasher, \& Grady, 
64

65

66

externally directed cognitive tasks, when more task-relevant networks (i.e., DAN) are recruited (Andrews-Hanna, Smallwood, \& Spreng, 2014). Importantly, the frontoparietal network (FPN) is thought to regulate the interaction between the DAN and DMN by switching these networks on and off to support cognition (Avelar-Pereira, Bäckman, Wåhlin, Nyberg, \& Salami, 2017; Dixon et al., 2017; Grady et al., 2016). Task-based and rsfMRI analyses have revealed that reduced DAN-DMN anticorrelations, possibly as a consequence of disrupted FPN engagement, is generally associated with worse cognitive performance and greater distractibility, particularly among older adults (Amer, Campbell, \& Hasher, 2016; Avelar-Pereira et al., 2017; Dixon et al., 2017; Esposito et al., 2018; Fox et al., 2005; Grady et al., 2016; Prakash, Heo, Voss, Patterson, \& Kramer, 2012; Sala-Llonch et al., 2012; Spreng et al., 2016).

This pattern of age-related increases in coupling of default and cognitive control networks is consistent with the default-executive coupling hypothesis of aging (DECHA; Turner \& Spreng, 2015), which is thought to reflect older adults' greater reliance on self-referential semantic knowledge to support cognitive performance in the face of age-related declines in fluid cognition (Spreng et al., 2018; Turner \& Spreng, 2015). Crucially, if access to semantic knowledge is relevant to the ongoing task, such as during creative thought, default-executive coupling may support task performance in older adults (Adnan, Beaty, Lam, Spreng, \& Turner, 2019; Spreng et al., 2014). Conversely, if retrieval of prior knowledge contributes to greater distractibility among participants, this pattern of defaultexecutive coupling either relates to worse cognitive performance, as confirmed by task-based analyses of cognitive domains assessing planning (Tower of London task; Turner \& Spreng, 2015), visuospatial judgment task, and fluid intelligence (Rieck, Rodrigue, Boylan, \& Kennedy, 2017), or greater semanticized recall of autobiographical memory (Spreng et al., 2018).

Network dynamics may further be modulated by the specific cognitive domain tested (Varangis, Habeck, \& Stern, 2021). For example, a recent study by Varangis and colleagues (2021) assessed participants' (20-80 years) performance across 11 in-scanner tasks of vocabulary, processing speed, 
fluid reasoning, and episodic memory. Functional connectivity was measured by calculating 1) average

90

correlations within-/between-networks, 2) segregation of the networks, and 3) global efficiency and modularity. Although older adults performed significantly worse than younger adults on the episodic

memory tasks, there was very little effect of age on functional connectivity measures during performance of these memory tasks. Instead, there were more robust effects of age on functional connectivity during participants' performance of the fluid reasoning tasks. Their findings highlight that the choice of cognitive task can have a significant impact on functional connectivity differences in aging. Taken together, although both task and rsfMRI connectivity studies have shown age-related dedifferentiation in intrinsic connectivity related to cognitive performance declines, functional connectivity may differ based on the specific task involved and how these networks dynamically adapt to accomplish these tasks. However, few studies have applied functional connectivity analysis to adult lifespan task fMRI data collected while participants encoded and retrieved episodic context memories, despite research indicating that associative context memory deficits are a hallmark of aging and emerge at midlife.

In addition, past task fMRI connectivity studies of episodic memory have assumed that agerelated differences in functional connectivity were the same in women and men, since data were not disaggregated by sex and/or gender. However, recent studies indicate that there may be sex differences in the effect of age on memory-related brain function (Rabipour, Rajagopal, Pasvanis, \& Rajah, 2021; Subramaniapillai et al., 2019). This is not surprising given that episodic memory deficits are observed in neurodegenerative diseases that exhibit sex differences in prevalence: women are disproportionately affected by Alzheimer's (Alzheimer's Association, 2020), while greater numbers of men have Parkinson's Disease (Cerri, Mus, \& Blandini, 2019). Sex differences in the prevalence of these diseases highlight the need to investigate the neural underpinnings of age-related episodic memory decline separately in women and men to inform the current models of healthy aging based on sex-aggregated 
113 data, and to provide important insight into sex-specific aging processes (Subramaniapillai, Almey,

114 Rajah, \& Einstein, 2021).

Previous studies have reported sex differences in rsfMRI intrinsic connectivity, with women

116 showing greater functional connectivity within the DMN (Allen et al., 2011; Bluhm et al., 2008; Filippi

117 et al., 2013; but see Weissman-Fogel, Moayedi, Taylor, Pope, \& Davis, 2010). In contrast, some

118 evidence points to greater connectivity in sensorimotor networks in men (Filippi et al., 2013; Allen et

119 al., 2011; but see Tomasi \& Volkow, 2012). One study reported sex differences in functional networks

120 across a wider age range of participants (18-65 years; Scheinost et al., 2015). Although both women

121 and men generally showed resting state functional connectivity decreases with age in the DMN, men showed greater age-related deficits in this network. In contrast, men and women showed divergent agerelated functional connectivity differences in the FPN (Scheinost et al., 2015). These sex differences in connectivity may act as important biomarkers for disorders such as Alzheimer's or Parkinson's Disease (Cerri et al., 2019; Damoiseaux et al., 2012), thus emphasizing the need to clarify sex-related differences in healthy aging to distinguish normal from atypical processes. and performance-related patterns of whole-brain activation across the adult lifespan (Ankudowich, Pasvanis, \& Rajah, 2017; Subramaniapillai et al., 2019). In brief, we found that across individuals, activity in regions of lateral PFC, parietal, and ventral visual cortex supported performance, but to a greater extent in young adulthood than in later stages (Ankudowich et al., 2017). Moreover, we found that when older individuals showed age-related increases in task-related activity across distributed areas of lateral PFC, parietal, and MTL, it did not necessarily support performance. More specifically,

134 during retrieval, age-related increases in hippocampal activity were related to worse memory

135 performance. When we explicitly investigated the role of sex differences on spatial context memory

136 performance in our adult lifespan cohort, we found that these generalized age-related increases and 
138 retrieval were specific to women (Subramaniapillai et al., 2019). Notably, despite there being sex

139 differences in the effect of age on memory-related brain activation, there were no behavioural sex

140 differences in context memory accuracy. Across studies, we concluded that our findings of more

141 diffuse, distributed task-related activity in older adulthood, particularly in women, was generally

142 consistent with the dedifferentiation theory of aging (Geerligs et al., 2015; Li, Lindenberger, \&

143 Sikström, 2001; Park et al., 2012; St-Laurent et al., 2011).

144 Whereas these previous studies undertaken in our lab have aimed to identify whole-brain

145 patterns of task-related activity that differ across the adult lifespan, the focus of the current study is to

146 understand how whole-brain patterns of task-related connectivity directly relate to context memory

147 performance across the adult lifespan. Although functional connectivity is typically assessed at rest, the

148 current study proposes a method of investigating functional connectivity, which examines task-related

149 functional connectivity after regressing the mean task-related activity from the fMRI signal to account

150 for task-timing-driven statistical associations (Cole et al., 2019). To derive independent effects of age

151 and performance, as these two variables might be confounded (Rugg, 2017), we orthogonalized the

152 continuous variables of age and retrieval accuracy. We then submitted these orthogonalized terms to a

153 multivariate PLS connectivity analysis to identify whole-brain patterns of connectivity amongst 216

154 parcellated regions based on the Power et al (2011) atlas during encoding and retrieval, maximally

155 relating age and accuracy on the spatial context memory task separately in women and men across the

156 adult lifespan.

157 The current methodological approach allows us to differentiate patterns of task-based memory-

158 related connectivity that reflect both age, sex, and performance effects in a data-driven way. With this

159 approach, we expect to find: i) similarities in performance-related task-based connectivity in both sexes

160 (Subramaniapillai et al., 2019), ii) age-related decreases in within-network connectivity and increases

161 in between-network connectivity that will be associated with poorer memory performance (Geerligs et

162 al., 2015), and indicative of a generalization and/or dedifferentiation of function in the aging brain ( $\mathrm{Li}$ 
163 et al., 2001), and iii) there will be sex differences in the networks exhibiting age-related increases in

164 between-network connectivity that relate to poorer memory performance. We also predict that there will be sex differences in age-related patterns of task-based functional connectivity that also support memory performance with advanced age, which may be indicative of compensation in the aging brain

167 (Subramaniapillai et al., 2019). Importantly, this investigation allows us to compare functional

168 activation at the brain regional level (Subramaniapillai et al., 2019) with respect to task-related

169 functional connectivity at the network level to determine whether these findings complement each 170 other at these different scales of analysis.

171

172

173

174

175

176

178

\section{Methods}

The original cohort consisted of 161 community-dwelling cognitively normal participants

between the ages of 19-76 years. We excluded middle-aged women who self-reported having irregular periods, symptoms of menopausal transition, and who had undergone hormone replacement therapy (HRT; $\mathrm{n}=20)$ since menopause transition and HRT influences memory-related brain activity (Henderson, 2010; R. Li, Cui, \& Shen, 2014; Rentz et al., 2017; Yonker et al., 2006). Our final cohort consisted of 141 participants $(65 \%$ women) between the ages of $19-76$ years $($ mean age $=47.11, \mathrm{SE}=$ 1.41 , mean education $=15.73$ years, $\mathrm{SE}=0.18)$. Participants reported no history of neurological or psychological illness, or serious cardiovascular disease. All participants were right-handed, as confirmed by the Edinburgh Inventory for Handedness.

Since this sample did not comprise a balanced sex ratio and was limited by the smaller proportion of men $(35 \%)$ who participated in the study, we used the cohort of 48 men and matched them with a cohort of women based on age and educational attainment to have a balanced sample of women and men. Closely matching women and men by their education level also accounts for its strong involvement as a reserve contributor in driving sex differences in performance (see Subramaniapillai et al., 2021 for gendered contribution of education as a reserve contributor). 
189 Therefore, this matched subsample consisted of 96 participants between the ages of 19-76 years (mean 190 age $=46.92[\mathrm{SE}, 1.77]$, mean education $=16$ years $[\mathrm{SE}, 2.0])$. This sample comprised 32 young adults

$191(19-35$ years, mean age $=26.34,[\mathrm{SE}, 0.56]$, mean education $=16.59[\mathrm{SE}, 0.26]$ years $), 32$ middle-aged

192 adults $(40-58$ years, mean age $=47.09$ [SE, 0.92], mean education $=15.63$ [SE, 0.32] years $)$, and 32

193 older adults $(60-76$ years, mean age $=67.34[\mathrm{SE}, 0.66]$, mean education $=15.78$ [SE, 0.42] years).

194 Participation in the study involved two separate testing sessions, conducted on different days.

195 During an initial session, participants underwent neuropsychological assessment (Mini-International

196 Neuropsychiatric Interview [MINI], inclusion cutoff $=/<2$; the Folstein Mini Mental State Examination

197 [MMSE], exclusion cutoff $<27$; the Beck Depression Inventory [BDI], exclusion cutoff $<15$;

198 California Verbal Learning Task [CVLT], exclusion cut-off based on recommendations by Norman,

199 Evans, Miller, \& Heaton (2000). Only participants who met the above neuropsychological criteria and 200 performed above chance on the practice context memory task returned to the testing center to complete

201 the fMRI scanning portion of the study. All participants gave their informed consent and were paid, and 202 the ethics board of the Faculty of Medicine at McGill University approved the study protocol.

203

204 Behavioural Methods

205 Stimuli and Procedure

The task stimulus set has been used in previous studies and has been independently rated for pleasantness (Kwon et al., 2016; Rajah, Languay, \& Valiquette, 2010). Stimuli consisted of black-andwhite photographs of faces that were varied in age and balanced for age and sex across experimental conditions. Each face presented during initial encoding was tested during subsequent retrieval, and 210 participants were scanned during both encoding and retrieval memory phases. A detailed description of

211 the task paradigm used in the current study can be found in previous studies from our lab (Ankudowich 212 et al., 2016, 2017). 
Using a mixed rapid event-related design, participants were scanned across 12 experimental

214 runs while they encoded and retrieved the spatial and temporal details of faces. Each run consisted of

an 'easy' temporal context memory task (TE) and an 'easy' spatial context memory task (SE), and

either a 'hard' temporal context memory task $(\mathrm{TH})$ or a 'hard' spatial context memory task (SH). Easy

and hard tasks differed in the number of stimuli to be encoded: 6 encoding stimuli for 'easy' tasks and

12 encoding stimuli for 'hard' tasks. Encoding was intentional, and at the start of each encoding phase,

participants were cued $(9 \mathrm{sec})$ to memorize either the spatial location (whether a face appeared on the

recently during encoding) of the faces and to the level of task difficulty. At encoding, each face was

presented $(2 \mathrm{sec})$ on either the left or the right of a central fixation cross. There was a variable inter-trial

interval (ITI) of $2.2-8.8 \mathrm{sec}$. During encoding, participants were instructed to rate the pleasantness of each face during encoding. Participants pressed a button with their right thumb to indicate a pleasant response and a button with their left thumb to indicate a neutral response using an MRI-compatible

fiber optic response box. Between encoding and retrieval memory phases, participants performed a

one-minute distractor task in which they were required to reverse alphabetize two words presented centrally on the computer screen. The distractor task was used to deter participants from actively rehearsing the encoding stimuli.

Following the distractor task, participants were presented with task instructions for retrieval (9 sec) to remind participants of the temporal and/or spatial context task demands. During retrieval, participants were presented with pairs of previously encoded faces for $6 \mathrm{sec}$. One of the faces was presented above a central fixation cross, and the other was presented below. During the easy versions of the retrieval task, participants viewed 3 pairs of faces, and during the hard versions of the retrieval task they viewed 6 pairs of faces. There was a variable ITI of $2.2-8.8 \mathrm{sec}$ between retrieval events.

236 For the spatial task, participants were asked to indicate which of the two faces was originally presented 
was originally presented $L E A S T / M O S T$ recently. Participants pressed a button under their right thumb

to indicate a face at the top of the screen and they pressed a button under their left thumb to indicate a

face at the bottom of the screen. Therefore, fMRI task-related activation was collected for 8 different

event-types in this experiment: encoding spatial easy (eSE), encoding temporal easy (eTE), encoding

spatial hard (eSH), encoding temporal hard $(\mathrm{eTH})$, retrieval spatial easy (rSE), retrieval temporal easy

(rTE), retrieval spatial hard $(\mathrm{rSH})$, retrieval temporal hard $(\mathrm{rTH})$.

\section{Task fMRI Imaging methods}

Structural and functional magnetic resonance imaging data were collected at the Douglas

Institute Brain Imaging Centre. Participants lied supine in a 3T Siemens Magnetom Trio scanner and

wore a standard 12-channel head coil. T1-weighted anatomical images were first acquired for each

participant at the start of the scanning session using a 3D gradient echo MPRAGE sequence $(\mathrm{TR}=$

$2300 \mathrm{msec}, \mathrm{TE}=2.98 \mathrm{msec}$, flip angle $=9^{\circ}, \mathrm{FOV}=256,1761 \mathrm{~mm}$ sagittal slices, $1 \times 1 \times 1 \mathrm{~mm}$

voxels). Blood-oxygen-level-dependent (BOLD) images were acquired with a single-shot T2*-

weighted gradient echo-planar imaging $(\mathrm{EPI})$ pulse sequence $(\mathrm{TR}=2000 \mathrm{msec}, \mathrm{TE}=30 \mathrm{msec}, \mathrm{FOV}=$

256 , matrix size $=64 \times 64$, in-plane resolution $4 \times 4 \mathrm{~mm}, 32$ oblique slices per whole-brain volume)

while participants performed the context memory tasks. Visual task stimuli were back-projected onto a

screen in the scanner bore using E-Prime software, and participants requiring correction for visual

acuity wore plastic corrective lenses. A variable ITI $(2.2-8.8 \mathrm{sec})$ was introduced to add jitter to

event-related acquisitions.

fMRI Basic Preprocessing

Reconstructed images were preprocessed in SPM version 8 software. The first 5 scans

261 (approximately 10 seconds) were discarded in order to allow for steady state magnetization of tissue.

262 For each participant, the origin of functional images was reoriented to the anterior commissure of that 
263 individual's acquired T1-weighted structural image. All functional images were then realigned to the 264 first image, and motion artifacts were corrected using a 6-degree rigid-body transformation (three 265 translation and three rotational parameters). Any experimental run in which within-run motion 266 exceeded $1.5 \mathrm{~mm}$ was excluded from analysis. Functional images were then normalized to an MNI EPI 267 template and resliced at $4 \times 4 \times 4 \mathrm{~mm}$ voxel resolution and smoothed with an $8 \mathrm{~mm}$ full-width at half 268 maximum (FWHM) isotropic Gaussian kernel. ArtRepair toolbox for SPM8 269 (http://cibsr.stanford.edu/tools/human-brain-project/artrepair- software.html) was used to correct slice 270 artifacts prior to realignment and volume artifacts after normalization and smoothing $(<5 \%$ interpolated data). Any run in which interpolated data exceeded 5\% was excluded from analysis.

\section{Analysis}

We focused our analysis on the behavioural and fMRI data collected during spatial context

sex differences in spatial episodic memory (Herlitz, Nilsson, \& Bäckman, 1997; A. Herlitz \&

Rehnman, 2008; Lewin, Wolgers, \& Herlitz, 2001). Furthermore, we were motivated to compare

current study findings using task functional connectivity with our published study investigating sex

differences and similarities in age-related and performance-related patterns of brain functional activity also using the same spatial context memory paradigm (Subramaniapillai et al., 2019).

\section{Behavioural Data Analysis}

Spatial Context Retrieval Accuracy and Reaction Time

Using R (R Core Team, 2013), we conducted robust linear mixed-effects regression (rlmer) 
model (Bates, Mächler, Bolker, \& Walker, 2015 for the lme4 package details) but additionally, it is

robust to outliers by down-weighting the impact of extreme measures on the model performance

(Koller 2016). The models contained the random effect of participants to account for the variability of

participants' performance between the easy and hard versions of the spatial context task. The models

used in terms of R syntax for spatial retrieval accuracy and reaction time, respectively, were:

Spatial Retrieval Accuracy $\sim$ Age x Sex x Task Difficulty + (1|Participant)

The continuous variable of age was standardized using a Z-score transformation, while the variables of

Image Analysis

\section{1 fMRI Preprocessing Steps}

1) Brain Parcellation

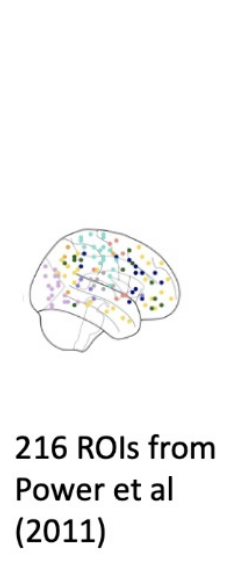

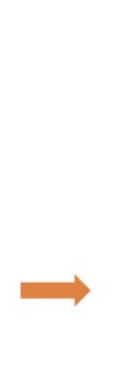

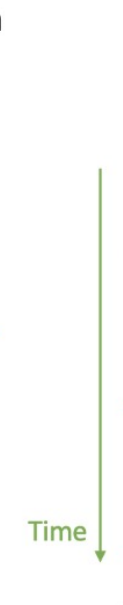

2) Signal Extraction
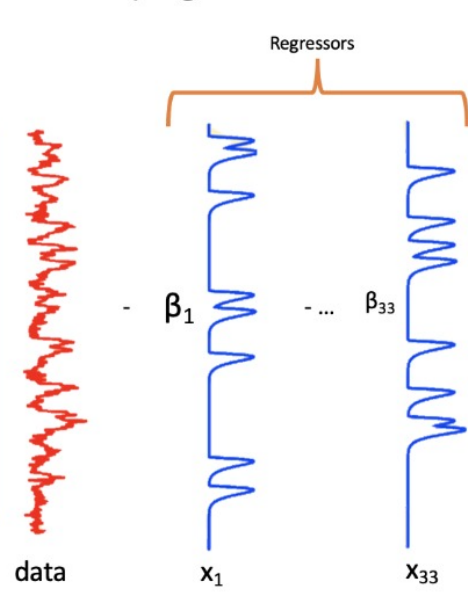

GLM to extract task residual signal (+ motion, WM \& CSF)

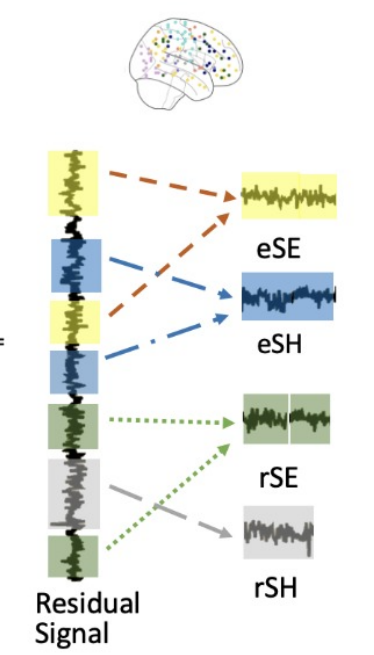

Generate one composite time series per task condition per ROI

Figure 1. The fMRI preprocessing steps involved (1) functional parcellation of each subject across the 216 unique ROIs from the Power et al. atlas; (2) applying a GLM to extract the task residual signal after regressing 33 regressors to generate one composite time series per task condition for each ROI; (3) generating four connectivity matrices for each task condition for every participant. 
302

303

304

305

306

307

308

309

310

311

312

313

314

315

316

317

318

319

320

321

322

323

324

325

326

fMRI Preprocessing for PLS Connectivity Analysis

\section{Brain Parcellation}

Using SPM's MarsBaR toolbox, the average time series for 264 regions of interest (ROIs)

defined by the Power et al. (2011) functional parcellation atlas were extracted for each subject for all task-related event-types across the full experiment. Each ROI was registered from the $2 \times 2 \times 2 \mathrm{~mm}^{3}$

Power atlas to the $4 \times 4 \times 4 \mathrm{~mm}^{3}$ voxel resolution of our functional scans. In order to do this, we took each ROI's central coordinates from the Power et al (2011) ROIs and identified a 7-voxel sphere surrounding the central coordinates. During this process of scaling down to the $4 \times 4 \times 4 \mathrm{~mm}^{3}$ voxel

resolution, we eliminated ROIs with voxels that were not common to all participants and/or overlapped with other ROIs. We also excluded cerebellar ROIs because our fMRI acquisition did not completely acquire these regions, and the uncertain network ROIs because they did not belong to a major functional system in the brain. We additionally combined the memory retrieval network with the default mode network because the few nodes belonging to the memory retrieval network are activated in cognitive functions (e.g., memory, imagination) commonly attributed to the default mode network (Huo, Li, Wang, Zheng, \& Li, 2018). Thus, we identified a total of 216 unique ROIs assigned to 9 brain networks: Auditory, Cingulo-opercular Task Control (CON), Default Mode Network (DMN), Dorsal Attention Network (DAN), Fronto-parietal Task Control Network (FPN), Salience, Sensory/Somatomotor Network (SSM), Visual Attention Network (VAN), Visual (see Supplementary Table 1 for list of coordinates and network affiliation). Figure 1 illustrates the preprocessing steps used to generate the connectivity matrices for participants across the four task conditions, which were subsequently submitted to the PLS analysis.

\section{fMRI Signal Extraction}

In order to examine task-related functional connectivity it is recommended that first the mean task/event-related activity across the full experiment be regressed out of the fMRI signal. This accounts 
327 for the confound of task-timing-driven statistical associations (Cole et al., 2019). To this aim, event-

328 related task activation for all 216 ROIs was estimated using SPM's General Linear Model (GLM) with

329 an ordinary least squares (OLS) approach (i.e., with AR(1) off), using a high pass filter set at 200 sec.

330 This GLM consisted of 12 task-related regressors: correct subsequent memory events for SE, SH, TE

331 and TH tasks (4), correct context retrieval events for SE, SH, TE, and TH tasks (4), incorrect

332 subsequent memory responses for all encoding tasks, incorrect context retrieval responses for all

333 retrieval tasks, encoding and retrieval task instructions, and distraction task. In addition, the 6

334 movement regressors generated by SPM during motion correction, the mean white matter and the

335 cerebrospinal fluid signals were also included as regressors in the GLM to correct for physiological

336 noise (Birn et al., 2014). Finally, the 12 temporal derivatives of the hemodynamic response function for

337 each of the task-related regressors and the constant (i.e., intercept) resulted in a total of 33 regressors

338 used in the GLM. Thus, this one GLM model was used to extract the mean residual time series for each

339 ROI per event-type using the MarsBaR toolbox in SPM (http://marsbar.sourceforge.net/). To allow

340 readers to compare our findings with and without regressing task-related activation, we re-ran our

341 analysis using connectivity matrices that did not regress mean task-related activity and found a similar

342 pattern of findings (Supplementary Figure 1).

343

344 Generating Functional Connectivity Matrices

345 Since the focus of our current analysis is the spatial version of the task, we only generated

346 functional connectivity matrices for each event-type of the spatial task. Each participant's residual time

347 series were concatenated across similar event-types in order to generate composite time series for each

348 event-type that roughly had the length of $\sim 30 \mathrm{sec}$, which has previously been shown to be reliably

349 sufficient for task-based connectivity analyses (e.g., Mohr et al., 2016). As a measure of functional

350 connectivity, Pearson correlations were conducted, and a correlation coefficient was computed for each

351 ROI with every other ROI. Connectivity matrices were created for each participant and event-type from 
352 the correlation coefficients, which then underwent Fisher z-transformation. Thus, in total, each subject

353 had four connectivity matrices, one for each of the four event-types (i.e., eSE, eSH, rSE, rSH) of size

$354216 \times 216$. Since the matrix is symmetrical around the diagonal, there were a total of 23,220 unique

355 connections or edges.

356

357

$\underline{\text { PLS Functional connectivity analysis }}$

Behavioural multivariate partial least squares (B-PLS) connectivity analysis was used to

identify patterns of task-based functional connectivity (Anthony R. McIntosh \& Mišić, 2013), due to its

ability to simultaneously detect distributed patterns of whole-brain connectivity that differ based on

participants' age, sex, and memory performance. Connectivity matrices for each individual were

362 organized by participant sex and task event-type and then stored in a data matrix that was stacked

363 similarly across participants. Since we were interested in how patterns of task-based functional

364 connectivity differed in women and men across the lifespan and related to memory performance on our

365 tasks, we conducted a PLS using participant age and retrieval accuracy as behavioural measures of

366 interest. We orthogonalized our behavioural vectors of age and accuracy to assess independent effects

367 of age and performance (consistent with Subramaniapillai et al., 2019; see also Ankudowich et al.,

368 2017). That is, outside of PLS, we conducted a regression analysis where task-specific retrieval

369 accuracy was used to predict age to obtain an age-residual vector that would be uncorrelated with

370 retrieval accuracy. These age-residual and retrieval accuracy vectors were then stacked in the same

371 manner as the fMRI data matrix by participant sex and by event-type. Given that the retrieval accuracy

372 behavioural vector did not have age regressed from it, it allowed us to assess connectivity associated

373 with age-related performance effects, whilst the age-residual allowed us to assess age effects

374 orthogonal to performance effects. The stacked fMRI data matrix was then cross-correlated with the

375 similarly stacked behavioural vectors. 
The resulting cross-correlation matrix was submitted to singular value decomposition (SVD).

377 SVD re-expresses the matrix as a set of orthogonal singular vectors or latent variables (LV). Each LV

378 consists of a singular value that reflects the proportion of matrix accounted for by that LV, and a pair of

379 vectors (a left singular vector consisting of the behavioural weights and a right singular vector

380 consisting of the connectivity weights) that reflect a symmetrical relationship between the pattern of

381 whole-brain connectivity and the experimental design/behaviour measures. The profile of behavioural

382 weights shows how the behavioural vectors of age and retrieval accuracy are correlated to the pattern of

383 whole-brain connectivity identified in the singular vector of connectivity weights. The connectivity

384 weights identify the collection of edges that, as a group, are maximally related to the behavioural 385 weights.

Significance testing for the LVs was done using 500 permutations $(p<0.05)$. The permutation

test assesses whether the functional networks and behavioural profiles are more strongly associated

with one another than expected by chance. Bootstrap resampling was performed to assess the reliability

of each of the edges (500 bootstraps, bootstrap ratio [BSR] threshold was set at 95 th percentile, $\mathrm{p}<$

0.001). Connectivity edge contribution was estimated with edge loadings, which is calculated as the

correlation of the participants' PLS-derived brain score pattern with their stacked connectivity

matrices. The pattern of edge loadings (i.e., correlations) is referred to as the loading matrix and

reflects whether edges are more positively or negatively associated with the behavioural weights. A

positive correlation coefficient in the loading matrix indicates a positive association with positive 
$\underline{\text { Post-Hoc Analyses }}$

We conducted two post-hoc analyses to determine whether findings from the primary matched sample analysis applied to the cohort of women that were not used (i.e., hold-out cohort; see

Supplementary Table 2 for the demographic summary of this cohort). First, we computed predicted

404 brain scores on the remaining cohort of women $(n=43)$ to determine the robustness and

405 generalizability of the primary PLS findings. Predicted brain scores were calculated by multiplying the

406 brain saliences of the primary analysis to the stacked connectivity matrices of the hold-out cohort of

407 women. We then computed an rlmer model to determine whether the brain scores of the hold-out

408 women were significantly different to the brain scores of the women in the primary analysis across the

409 three significant LVs. The model used in terms of R syntax was:

$$
\operatorname{rlmer}(\mathrm{LV} \sim \text { Data_Source+ }(1 \mid \mathrm{ID})+(1 \mid \text { Task_EventType }))
$$

413 Three models were run in total, one for each of the three significant LVs. The Data_Source was

414 whether women were from the original 'matched' cohort or the 'holdout' cohort. The Task_EventType 415 represents the four task event-types of the study (eSE, eSH, rSE, rSH). No significant difference in 416 brain scores between the groups would confirm that the brain-behaviour findings correspond to the 417 cohort of women excluded from the original age- and education-matched cohort.

418 In our second post-hoc analysis, we ran a within-sex partial least squares analysis using the full 419 cohort of women from the primary and hold-out cohorts $(n=91)$ to determine whether the within-sex 420 PLS findings were similar to the primary between-sex PLS findings (see Supplementary Table 3 for 421 demographic summary of this cohort of women).

422 Since our findings could be driven by sex differences in intracranial volume (ICV) with men 423 having larger ICV than women, we ran a supplementary analysis using a sub-cohort $(\mathrm{n}=50)$ of women 
424 and men matched according to age, education, and ICV to determine whether this pattern of LV

425 findings were similar to the primary analysis (see Supplementary Figure 2).

426

\section{Results}

428

429

\section{Behavioural Results}

Tables 1 and 2 summarizes the participant demographic and neuropsychological information across the age groups for the full $(n=141)$ and matched cohort $(n=96)$, respectively. Within the matched cohort, post hoc analyses revealed no significant differences in age and education between women and men within each age group ( $\mathrm{p}>.05)$, confirming the successful age- and sex-matching of participants. Behaviourally, the rlmer model investigating the effects of age, sex, and task difficulty on memory accuracy showed a main effect of age $(\beta=-0.03[\mathrm{SE}, 0.01] ; \mathrm{t}=-2.35, \mathrm{p}<.05)$ and task difficulty $(\beta=$ -

$0.04[\mathrm{SE}, 0.01] ; \mathrm{t}=-3.00, \mathrm{p}<.05)$. Younger adults had greater accuracy than older adults on the tasks, and generally, participants performed worse on the SH task compared to the SE task. No other main effects or interactions were significant. task difficulty $(\beta=130.23,[\mathrm{SE}=36.71] ; \mathrm{t}=3.55, \mathrm{p}<.05)$ on reaction time. Young adults were faster

442 than older adults across SE and SH tasks, and participants took longer to respond to the SH task than

443 the SE task. No other main effects or interactions were significant. The behavioural results for the spatial task results were consistent between the full and matched cohorts. 
bioRxiv preprint doi: https://doi.org/10.1101/2021.07.27.453878; this version posted July 27, 2021. The copyright holder for this preprint (which was not certified by peer review) is the author/funder. All rights reserved. No reuse allowed without permission.

Table 1. Mean Demographic and Behavioural Measures (and Standard Errors) according to Age Group and Sex $(\mathrm{N}=141)$

\begin{tabular}{|c|c|c|c|c|c|c|c|}
\hline & \multirow{2}{*}{\multicolumn{2}{|c|}{ Younger Adults (YA) }} & \multirow{2}{*}{\multicolumn{2}{|c|}{ Middle Aged Adults (MA) }} & \multirow{2}{*}{\multicolumn{2}{|c|}{ Older Adults (OA) }} & \multirow[b]{3}{*}{$p$} \\
\hline & & & & & & & \\
\hline & Males & Females & Males & Females & Males & Females & \\
\hline Sample size (n) & 16 & 29 & 17 & 35 & 16 & 28 & \\
\hline Age (years) & $26.56(0.84)$ & $25.90(0.72)$ & $47.18(1.31)$ & $49.69(0.90)$ & $67.13(1.04)$ & $66.14(0.65)$ & \\
\hline Educations (years) & $16.44(0.34)$ & $15.90(0.42)$ & $15.59(0.41)$ & $15.66(0.36)$ & $16.19(0.61)$ & $15.07(0.42)$ & ns \\
\hline Predicted full-scale IQ & $119.72(1.34)$ & $118.84(1.02)$ & $\begin{array}{l}119.54 \\
(1.43)\end{array}$ & $119.44(0.85)$ & $119.73(1.04)$ & $120.03(1.06)$ & $\mathrm{ns}$ \\
\hline BDI & $4.06(0.87)$ & $3.32(0.74)^{*}$ & $3.12(0.87)$ & $4.54(0.72)$ & $4.38(1.04)$ & $3.79(0.57)$ & ns \\
\hline CVLT-LFR & $13.25(0.48)$ & $14.14(0.28)$ & $11.82(0.76)$ & $13.46(0.27)$ & $12.00(0.56)$ & $13.25(0.44)$ & $\begin{array}{l}\mathrm{p}< \\
.05^{\% \#}\end{array}$ \\
\hline CVLT-LCR & $13.38(0.41)$ & $\begin{array}{l}14.24(0.33) \\
15.55\end{array}$ & $12.41(0.62)$ & $13.71(0.30)$ & $12.50(0.47)$ & $13.39(0.39)$ & $\begin{array}{l}\mathrm{p}< \\
.05^{\% \# \#}\end{array}$ \\
\hline CVLT-RG & $15.38(0.18)$ & $(0.13)$ & $15.35(0.21)$ & $15.34(0.15)$ & $15.13(0.18)$ & $15.20(0.17)$ & ns \\
\hline BMI $\left(\mathrm{kg} / \mathrm{m}^{2}\right)$ & $23.93(0.65)$ & $22.03(0.68)$ & $25.15(0.78)$ & $24.60(0.67)$ & $24.37(0.57)$ & $25.80(0.72)^{*}$ & ns \\
\hline $\begin{array}{l}\text { SE retrieval accuracy } \\
\text { (\%correct) }\end{array}$ & $0.85(0.03)$ & $0.90(0.01)$ & $0.88(0.02)$ & $0.86(0.02)$ & $0.80(0.02)$ & $0.83(0.02)$ & $\mathrm{p}<.01^{\#}$ \\
\hline $\begin{array}{l}\text { SH retrieval accuracy } \\
\text { (\%correct) }\end{array}$ & $0.84(0.03)$ & $0.89(0.09)$ & $0.83(0.03)$ & $0.84(0.02)$ & $0.73(0.03)$ & $0.78(0.02)$ & $\mathrm{p}<.01^{\#}$ \\
\hline SE retrieval RT (msec) & $\begin{array}{l}2270.69 \\
(101.68)\end{array}$ & $\begin{array}{l}2194.72 \\
(104.90)\end{array}$ & $\begin{array}{l}2353.35 \\
(122.95)\end{array}$ & $\begin{array}{c}2464.396 \\
(93.95)\end{array}$ & $\begin{array}{l}2633.79 \\
(138.26)\end{array}$ & $\begin{array}{c}2878.14 \\
(83.98)\end{array}$ & $\mathrm{p}<.01^{\#}$ \\
\hline SH retrieval RT (msec) & $\begin{array}{c}2422.47 \\
(96.33)\end{array}$ & $\begin{array}{l}2267.92 \\
(100.09)\end{array}$ & $\begin{array}{l}2467.69 \\
(109.12)\end{array}$ & $2610.75(77.30)$ & $\begin{array}{l}2765.84 \\
(154.00)\end{array}$ & $\begin{array}{l}2871.45 \\
(82.69)\end{array}$ & $\mathrm{p}<.01^{\#}$ \\
\hline
\end{tabular}

*One participant had missing BDI $(\mathrm{n}=28)$ or BMI $(\mathrm{n}=29)$ information. Values in brackets represent the standard error. A linear regression of Age $x$ Sex was performed on each of the measures (significance of $p<.05$ used). \% The linear regression produced a significant effect of Sex, such that women outperformed men on this score. \# The linear regression produced a significant main effect of Age. The fMRI behavioural measures revealed that OA participants performed significantly worse than younger and middle-aged participants and with significantly greater RT to complete the spatial tasks. BDI = Beck Depression Inventory; LFR = Long-form Free Recall; LCR = Long-form Cued Recall; RG = Recognition; BMI = body mass index. 
bioRxiv preprint doi: https://doi.org/10.1101/2021.07.27.453878; this version posted July 27, 2021. The copyright holder for this preprint (which was not certified by peer review) is the author/funder. All rights reserved. No reuse allowed without permission.

Table 2. Mean Demographic and Behavioural Measures (and Standard Errors) according to Age Group and Sex $(\mathrm{N}=96)$

\begin{tabular}{|c|c|c|c|c|c|c|c|}
\hline & \multicolumn{2}{|c|}{ Younger Adults (YA) } & \multicolumn{2}{|c|}{ Middle Aged Adults (MA) } & \multicolumn{2}{|c|}{ Older Adults (OA) } & \multirow[b]{2}{*}{$p$} \\
\hline & Males & Females & Males & Females & Males & Females & \\
\hline$\overline{\text { Sample size (n) }}$ & 16 & 16 & 16 & 16 & 16 & 16 & \\
\hline Age (years) & $26.56(0.84)$ & $26.13(0.76)$ & $46.94(1.37)$ & $47.25(1.28)$ & $67.13(1.04)$ & $67.56(0.85)$ & \\
\hline Educations (years) & $16.44(0.34)$ & $16.75(0.40)$ & $15.44(0.41)$ & $15.81(0.49)$ & $16.19(0.61)$ & $15.38(0.57)$ & ns \\
\hline Predicted full-scale IQ & $119.72(1.34)$ & $118.49(1.33)$ & $120.42(1.21)$ & $120.76(1.25)$ & $119.73(1.04)$ & $120.65(1.08)$ & ns \\
\hline BDI & $4.06(0.87)$ & $3.53(1.08)^{*}$ & $3.25(0.92)$ & $4.31(1.12)$ & $4.38(1.04)$ & $3.25(0.73)$ & ns \\
\hline CVLT-LFR & $13.25(0.48)$ & $13.50(0.41)$ & $11.81(0.81)$ & $13.19(0.43)$ & $12.00(0.56)$ & $13.19(0.56)$ & $\mathrm{p}<.05 \%$ \\
\hline CVLT-LCR & $13.38(0.41)$ & $13.69(0.45)$ & $12.44(0.66)$ & $13.44(0.51)$ & $12.50(0.47)$ & $13.25(0.46)$ & ns \\
\hline CVLT-RG & $15.38(0.18)$ & $15.31(0.20)$ & $15.44(0.20)$ & $15.31(0.24)$ & $15.13(0.18)$ & $14.94(0.23)$ & ns \\
\hline $\operatorname{BMI}\left(\mathrm{kg} / \mathrm{m}^{2}\right)$ & $23.93(0.65)$ & $22.01(0.70)$ & $24.85(0.76)$ & $23.63(0.78)$ & $24.37(0.57)$ & $25.10(0.86)$ & $\mathrm{p}<0.05^{\prime}$ \\
\hline SE retrieval accuracy (\%correct) & $0.85(0.03)$ & $0.90(0.02)$ & $0.88(0.02)$ & $0.87(0.02)$ & $0.80(0.02)$ & $0.84(0.02)$ & $\mathrm{p}<.01^{\#}$ \\
\hline SH retrieval accuracy (\%correct) & $0.84(0.03)$ & $0.89(0.03)$ & $0.83(0.03)$ & $0.86(0.02)$ & $0.73(0.03)$ & $0.78(0.02)$ & $\mathrm{p}<.01^{\#}$ \\
\hline SE retrieval RT (msec) & $2270.69(101.68)$ & $\begin{array}{l}2105.18 \\
(141.44)\end{array}$ & $\begin{array}{l}2393.30 \\
(123.78)\end{array}$ & $\begin{array}{l}2448.30 \\
(160.42)\end{array}$ & $\begin{array}{l}2633.79 \\
(138.26)\end{array}$ & $2894.03(92.98)$ & ) $<<.01^{\#}$ \\
\hline$\underline{\mathrm{SH}}$ retrieval RT (msec) & $\begin{array}{r}2422.47 \\
(96.33)\end{array}$ & $\begin{array}{r}2218.69 \\
(144.57) \\
\end{array}$ & $\begin{array}{r}2514.95 \\
(104.71) \\
\end{array}$ & $\begin{array}{l}2596.62 \\
(132.19)\end{array}$ & $2765.84(154.00)$ & $2874.13(93.05)$ & $\underline{\mathrm{p}<.01^{\#}}$ \\
\hline
\end{tabular}

*One participant had missing BDI information $(\mathrm{n}=15)$. Values in brackets represent the standard error. A linear regression of Age $x$ Sex was performed on each of the measures (significance of $\mathrm{p}<.05$ used). $\%$ The linear regression produced a significant effect of Sex, such that women outperformed men on this score. ${ }^{\wedge}$ Age $\mathrm{x}$ Sex interaction of BMI: age-related increase in BMI; younger and middle-aged adult men had higher BMI than their female counterparts; and older men had higher BMI than older women. \# The linear regression produced a significant main effect of Age. The fMRI behavioural measures revealed that OA participants performed significantly worse than younger and middle-aged participants and with significantly greater RT to complete the spatial tasks. BDI = Beck Depression Inventory; LFR = Long-form Free Recall; $\mathrm{LCR}=$ Long-form Cued Recall; $\mathrm{RG}=$ Recognition; $\mathrm{BMI}=$ body mass index.

\section{Functional connectivity results}

The PLS analysis of the fMRI data identified three significant LVs at $\mathrm{p}<0.05$. LV1 (42\%

447 cross-block covariance) identified sex differences in how age impacted memory-related brain

448 connectivity and how brain connectivity related to memory accuracy. LV2 and LV3 (26.50\% and

$4499.41 \%$ cross-block covariance respectively) demonstrated sex similarities in how brain connectivity

450 related to memory performance but differences in how age impacted memory-related brain

451 connectivity. These findings were consistent with PLS findings that matched women and men by ICV

452 (Supplementary Figure 2).

Figures 2-4 depict the relevant LV information for LVs 1-3, respectively. The subplots include

454 the 1) behavioural weights, 2) thresholded loading matrix, 3) network density matrix, and the 4) brain

455 figure representing the highly involved nodes. The behavioural weights (1) indicate how the loading 
matrix relates to the behavioural vectors of age and accuracy in women and men. The thresholded connectivity matrix (2) represents the 95th percentile of the z-score values of correlation coefficients.

The network density matrix (3) represents the sum of the unthresholded significant edges divided by generated two density plots because calculations were done separately on the positive and negative correlation coefficients. LV1 and LV2 did not produce any significant negative correlations, so only the network density matrix for the negative correlations in LV3 is presented (Figure 4D). Finally, the brain

determined by the ranked sum of the correlation values from most to least involved. the full cohort of women that were marginal in the primary analysis. Since the behavioural weights of LV2 was identical to LV3 of the primary analysis, we present LV2 findings of this within-sex analysis in Supplementary Figure 3.

\section{LV1: Sex differences in age- and accuracy-related task connectivity}


481 correlations with the SSM, Salience, and CON networks, in addition to within-network connectivity.

482 The loading matrix (Figure 2C) and behavioural weights (Figure 2A) together indicate that in women, 483 this pattern of positive brain connectivity during encoding and retrieval was negatively correlated with 484 age and was positively correlated with memory performance for the SH task. Of note, women showed a 485 trending effect of memory-related brain connectivity with task performance in the original matched 486 analysis, which was significant in the larger within-sex analysis of women (Figure 2B). Conversely, 487 men did not show any age-related differences in the pattern of task-based connectivity identified in 488 LV1. In addition, in men, increased positive connectivity in these same networks during encoding and 489 retrieval, was related to poorer memory performance across all tasks. The network density matrix 490 (Figure 2D) and brain figure (Figure 2E) corroborate the loading matrix findings. The thresholded 491 connectivity matrix representing the $95^{\text {th }}$ percentile of the $\mathrm{z}$-score values of correlation coefficients for 492 LV1 (Figure 2C) is available online https://github.com/sivaniya/M48F48_conn_matrix. 


\section{LV1: Sex differences in age- and accuracy-related task connectivity}

A
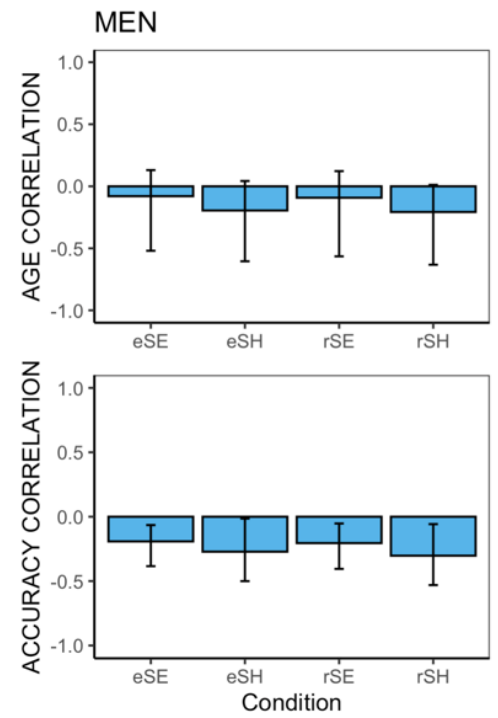

B
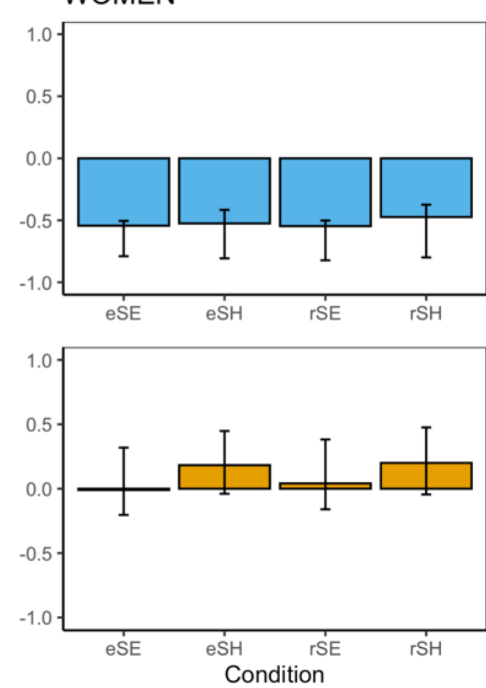

$\mathrm{D}$

$\mathrm{C}$

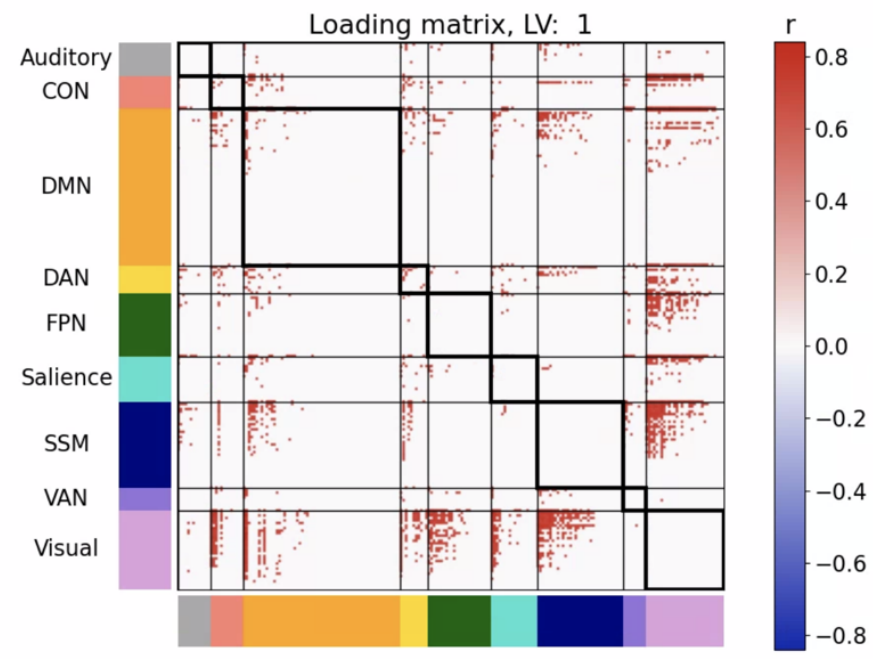

$\mathrm{E}$
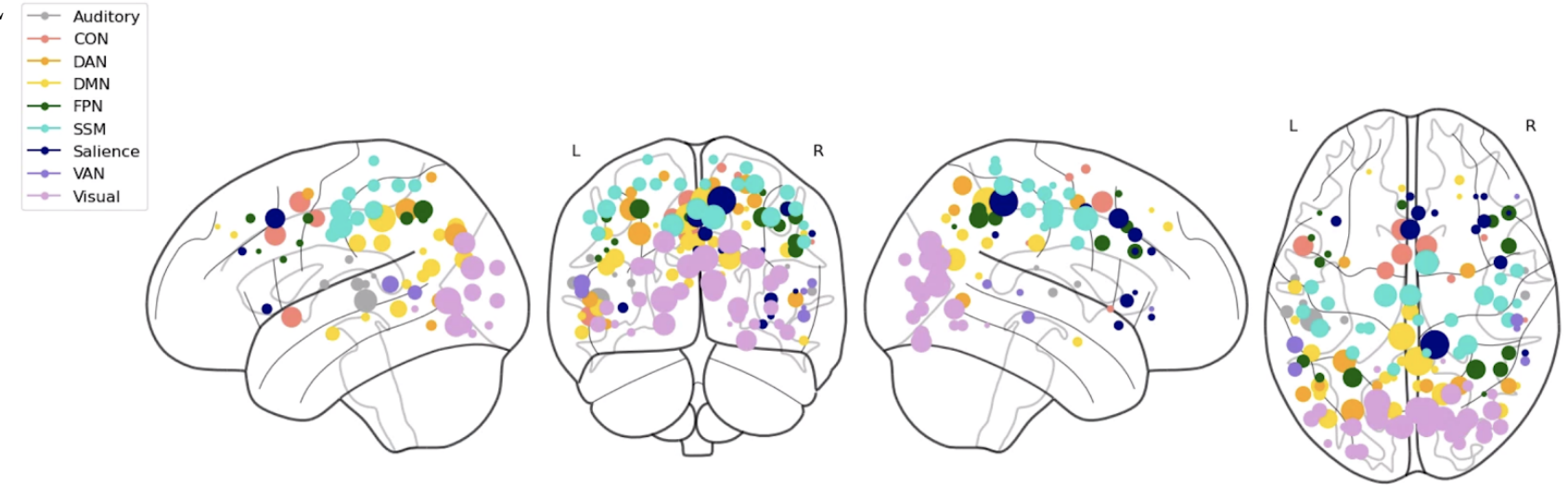

Figure 2. LV1 reflects sex differences in age- and memory-related brain connectivity. Correlation between the behavioural 
vectors of age and accuracy with the task fMRI connectivity of participants for the (A) primary analysis and (B) within-sex sample (LV1). Error bars represent bootstrapped standard deviations. (C) Thresholded 95th percentile of correlations between participants' task fMRI data and behavioural profile indicated in A. (D) The density plot for the positive correlation coefficients (i.e., sum of the significant correlation coefficients after thresholding, divided by the total number of edges between any two networks). The density matrix for the negative correlation coefficients is not presented because there were no significant edges. (E) Most densely connected nodes from the positive salience loading matrix as represented by the rank sum of the correlation coefficients of the thresholded matrix. Greater node size represents greater node involvement. eSE $=$ encoding spatial easy; $\mathrm{eSH}=$ encoding spatial hard; $\mathrm{rSE}=$ retrieval spatial easy; $\mathrm{rSH}=$ retrieval spatial hard; $\mathrm{CON}=$ cingulo-opercular network; DMN = default mode network; DAN = dorsal attention network; FPN = frontoparietal network; $\mathrm{SSM}=$ somatomotor network; VAN $=$ ventral attention network. significant positive correlation coefficients amongst most networks, with few significant correlations

(Figures 3B and 3C) indicates there were a significant number of positive correlations between the

demonstrate that there were age-related increases in task-related connectivity in the aforementioned 
bioRxiv preprint doi: https://doi.org/10.1101/2021.07.27.453878; this version posted July 27, 2021. The copyright holder for this preprint (which was not certified by peer review) is the author/funder. All rights reserved. No reuse allowed without permission.

\section{LV2: Sex differences in age-, but not accuracy-, related task connectivity}

A
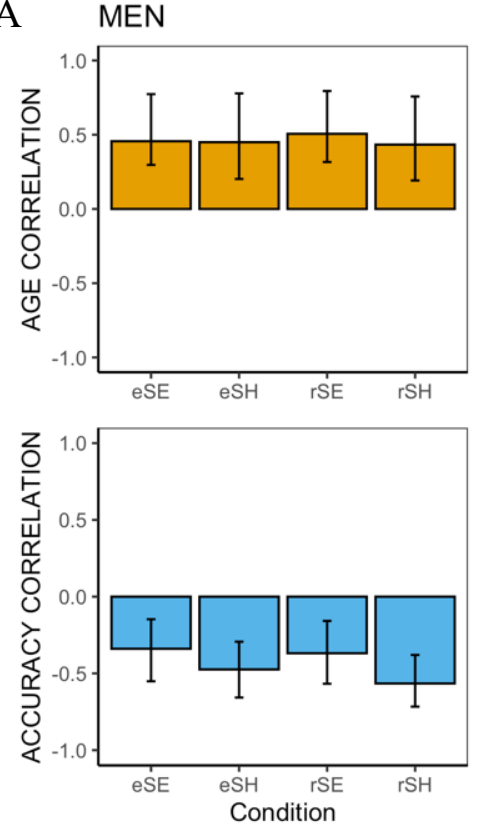

WOMEN
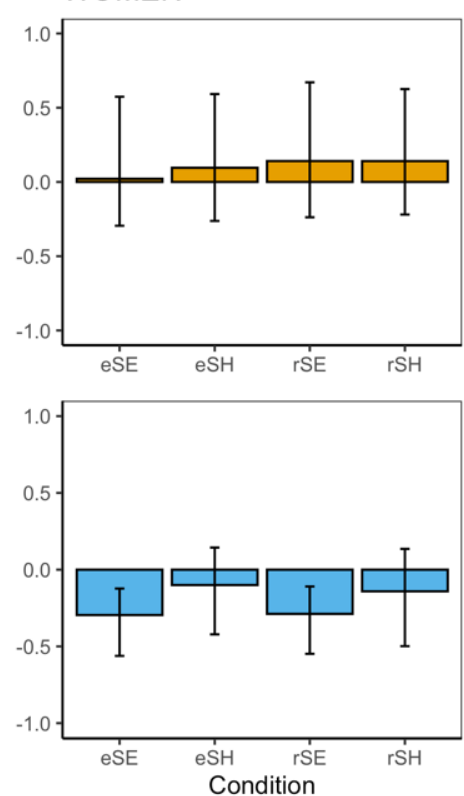

B

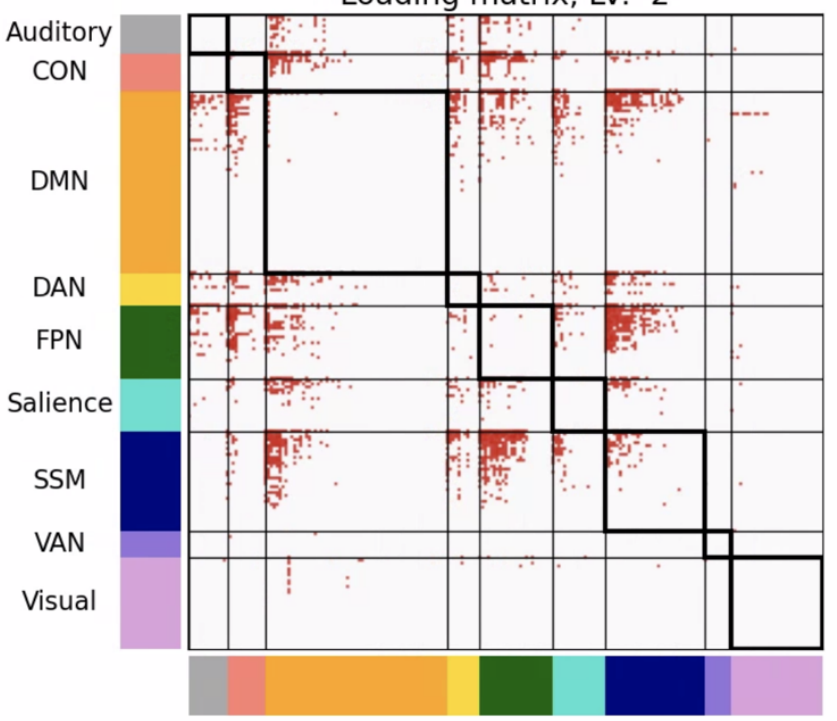

0.6

$-0.4$

$-0.2$

$-0.0$

$-0.2$

$-0.4$

$-0.6$

$\mathrm{C}$

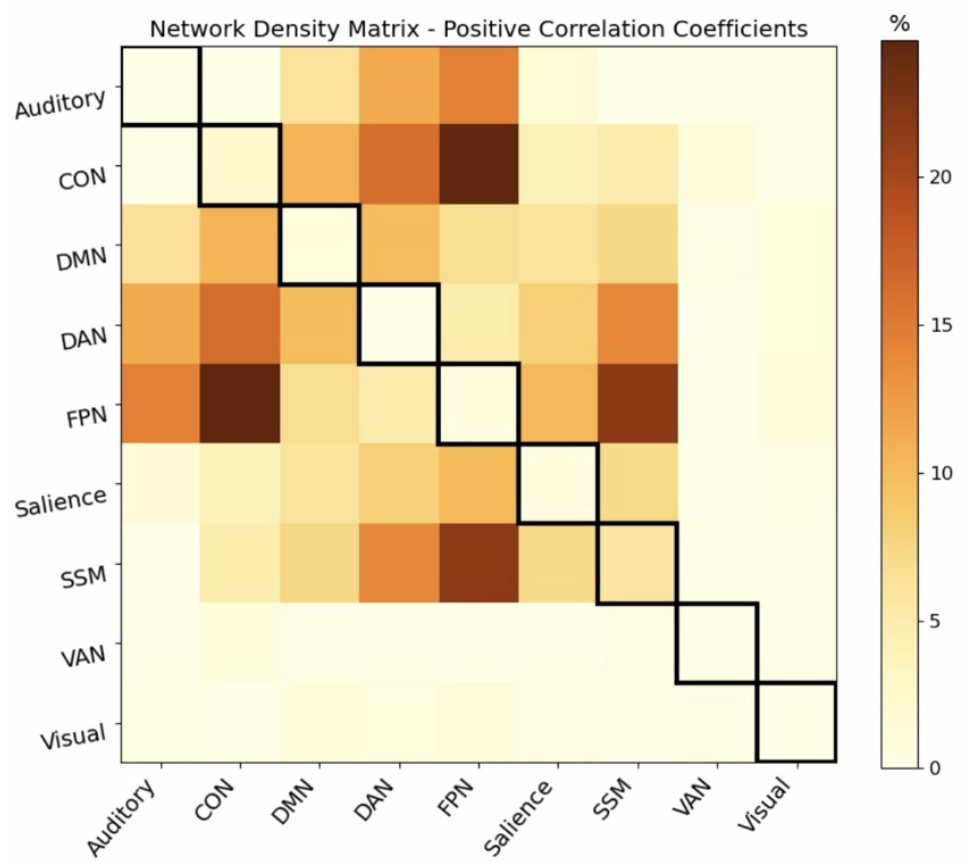

D
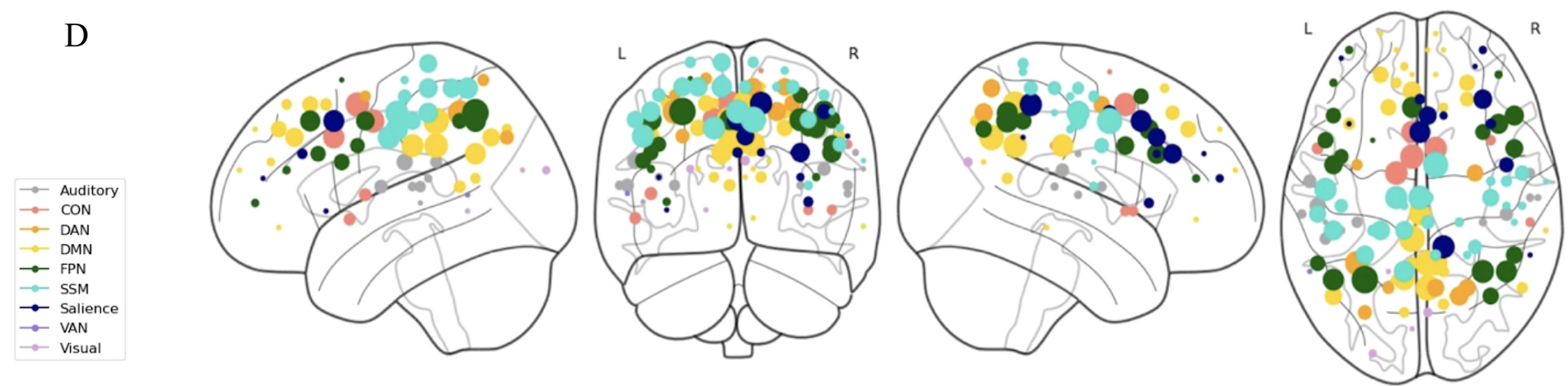
532

Figure 3. LV2 reflects sex similarities in memory-related brain connectivity, but differences in age-related brain connectivity. (A) Correlation between the behavioural vectors of age and accuracy with the task fMRI connectivity of participants. Error bars represent bootstrapped standard deviations. (B) Thresholded 95th percentile of correlations between participants' task fMRI data and behavioural profile indicated in A. (C) The density plot for the positive correlation coefficients (i.e., sum of the significant correlation coefficients after thresholding, divided by the total number of edges between any two networks). The density matrix for the negative correlation coefficients is not presented because there were no significant edges. (D) Most densely connected nodes from the positive salience loading matrix as represented by the rank sum of the correlation coefficients of the thresholded matrix. Greater node size represents greater node involvement. eSE $=$ encoding spatial easy; eSH = encoding spatial hard; $\mathrm{rSE}=$ retrieval spatial easy; $\mathrm{rSH}=$ retrieval spatial hard; $\mathrm{CON}=$ cingulo-opercular network; DMN = default mode network; DAN = dorsal attention network; FPN = frontoparietal network; $\mathrm{SSM}=$ somatomotor network; VAN = ventral attention network.

\section{LV3: Sex similarities in accuracy-related, but differences in age-related, task connectivity}

LV3 accounted for $9.41 \%$ of the cross-block covariance and identified significant positive

correlation coefficients between several networks. The loading and density matrices (Figure 4B and

4C) indicate that there was greatest density of positive correlations between the FPN and Salience

networks, and between the Visual network and VAN. In addition, there were dense within-network

positive correlations in the Salience, VAN, and Visual networks. The loading matrix (Figure 4B) and

behavioural weights (Figure 4A) indicate that in men, age was negatively correlated with increased

connectivity in the aforementioned networks, and memory performance during SE tasks was also

negatively correlated with increased functional connectivity in these networks. In women, age was not

significantly correlated with connectivity amongst these networks, and memory performance for both

SE and SH tasks was negatively correlated with increased functional connectivity in these networks.

LV3 also identified negative correlations within DAN and between DAN, FPN and Salience networks;

and, between Visual-FPN and Salience, and VAN-FPN (Figure 4D). These patterns of within- and

between-network connectivity were correlated with advanced age in men, better memory performance

during SE tasks for men, and better memory performance during both tasks in women. Thus, both LV2

and LV3 demonstrated similar performance effects in both sexes but identified age-effects in only men.

Thus, both LV2 and LV3 demonstrated similar performance effects in both sexes but identified age-

effects in only men. The thresholded connectivity matrix representing the $95^{\text {th }}$ percentile of the $\mathrm{z}$-score

values of correlation coefficients for LV3 (Figure 4B) is available online

https://github.com/sivaniya/M48F48_conn_matrix. 
LV3: Sex similarities in accuracy-related, but differences in age-related, task connectivity

A
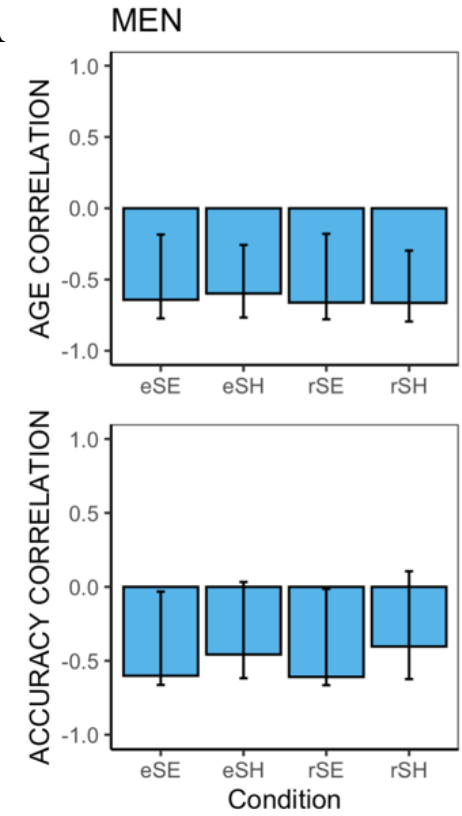

$\mathrm{C}$

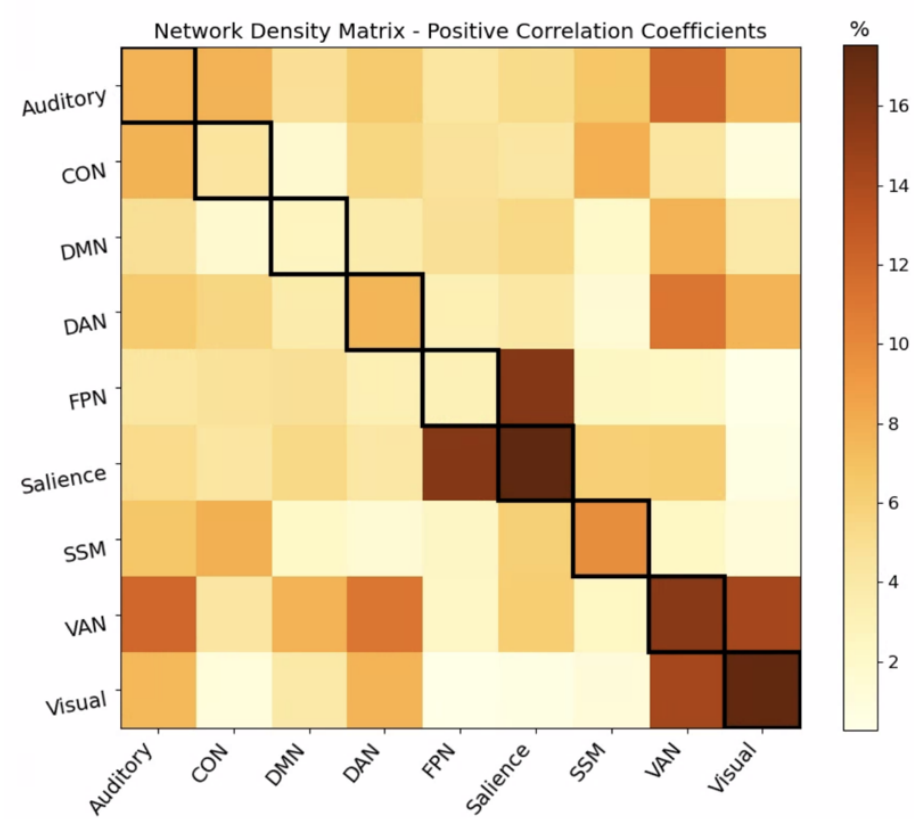

WOMEN
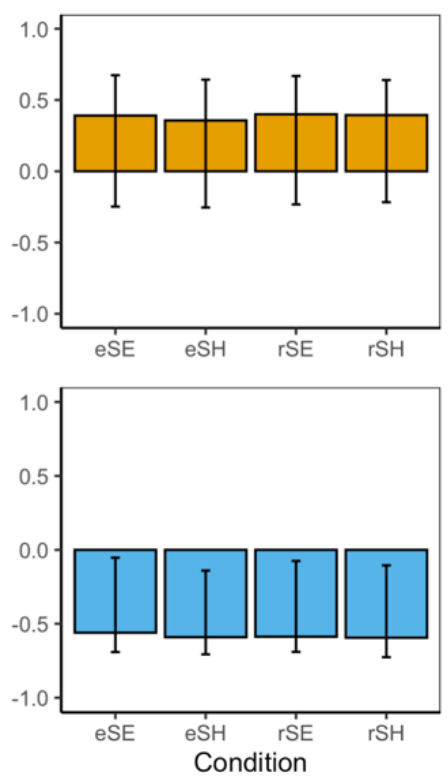

B

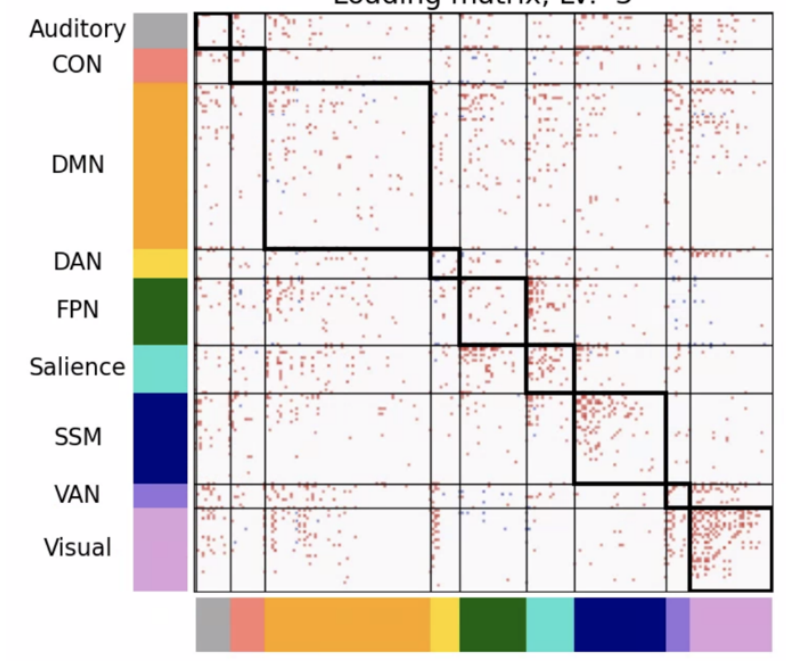

$\mathrm{D}$

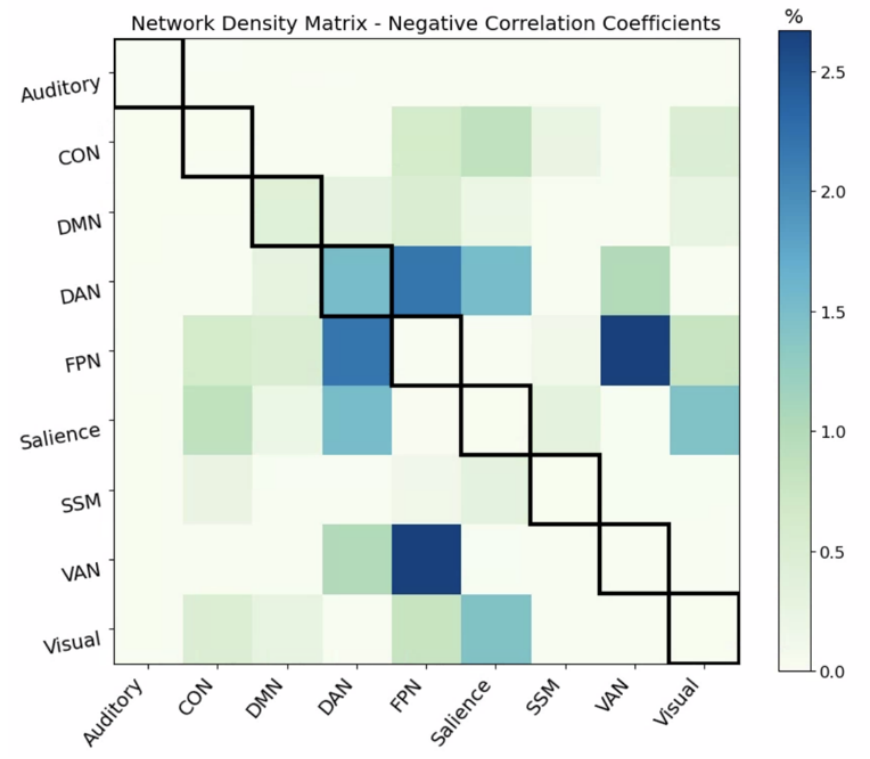

E

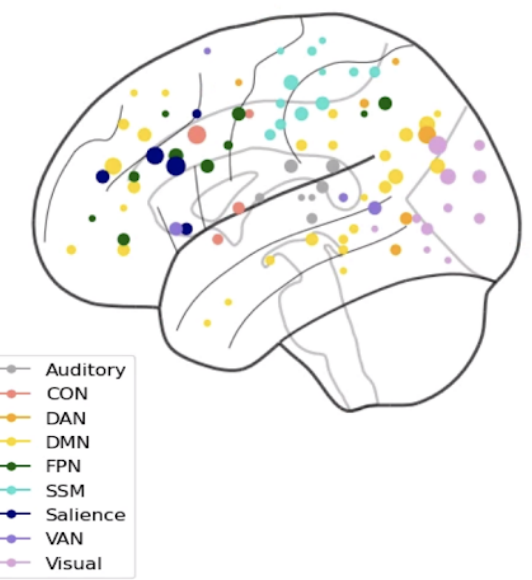

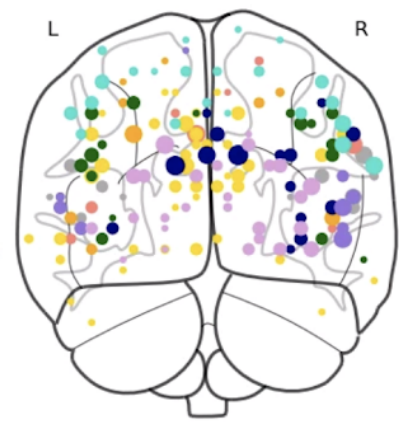
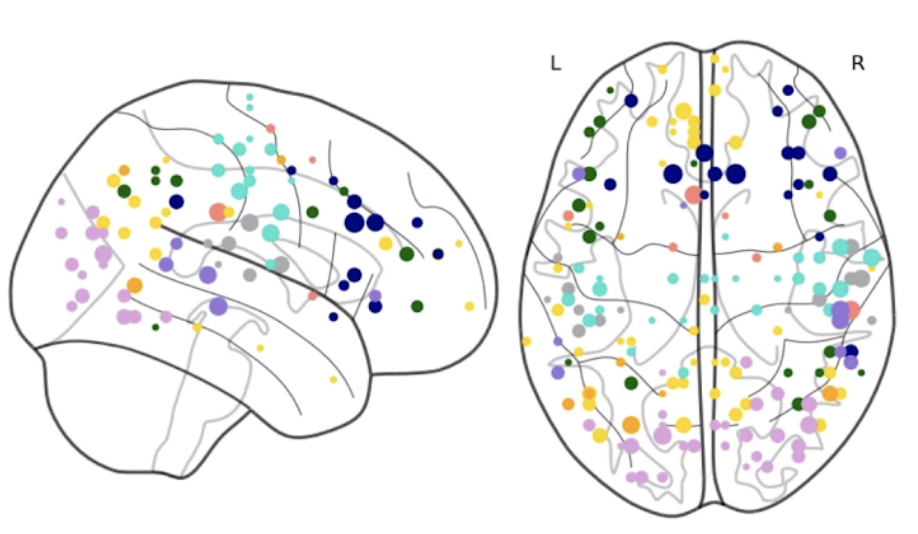
Figure 4. LV3 reflects sex similarities in memory-related brain connectivity, but differences in age-related brain connectivity. (A) Correlation between the behavioural vectors of age and accuracy with the task fMRI connectivity of participants. Error bars represent bootstrapped standard deviations. (B) Thresholded 95th percentile of correlations between participants' task fMRI data and behavioural profile indicated in A. (C) The density plot for the positive correlation coefficients (i.e., sum of the significant correlation coefficients after thresholding, divided by the total number of edges between any two networks). (D) The density matrix for the negative correlation coefficients. (E) Most densely connected nodes from the positive salience loading matrix as represented by the rank sum of the correlation coefficients of the thresholded matrix. Greater node size represents greater node involvement. eSE = encoding spatial easy; eSH = encoding spatial hard; $\mathrm{rSE}=$ retrieval spatial easy; $\mathrm{rSH}=$ retrieval spatial hard; $\mathrm{CON}=$ cingulo-opercular network; $\mathrm{DMN}=$ default mode network; DAN = dorsal attention network; FPN = frontoparietal network; SSM = somatomotor network; VAN = ventral attention network.

553

554

555

\section{Discussion}

The current study investigated sex differences in the effects of age and retrieval accuracy (memory performance) on memory-related task functional connectivity. The behavioural results were consistent with our prior findings (Ankudowich, Pasvanis, \& Rajah, 2017; Subramaniapillai et al., 2019). We found significant main effects of age and task difficulty on spatial context memory accuracy and reaction time. Older adults performed worse than younger adults on retrieval accuracy and experienced slower processing speeds to complete the spatial context tasks. A main effect of task difficulty showed that participants, independent of age, generally performed worse on the spatial hard context tasks than the easy version of the task. Moreover, we found no significant effect of sex on accuracy and reaction time, nor any significant interactions of age and sex. Therefore, the sample included in this connectivity analysis parallels that used in our prior study examining sex differences in the effect of age and memory performance on spatial context memory activation (Subramaniapillai et al., 2019). These behavioural findings counter some prior work indicating women and men differ on episodic memory (Astur, Purton, Zaniewski, Cimadevilla, \& Markus, 2016; Bremner et al., 2001; Keightley, Winocur, Burianova, Hongwanishkul, \& Grady, 2006; Ragland, Coleman, Gur, Glahn, \& Gur, 2000). However, closer examination of studies revealing sex differences in episodic memory demonstrate considerable range of outcomes even within-sex, with small to medium effect sizes across the studies (Asperholm, Van Leuven, \& Herlitz, 2020; Herlitz \& Rehnman, 2008). Thus, straying away from average-based group analyses and towards understanding individual differences may be a more 
572 fruitful approach that appropriately considers the range of diversity observed within- and between-

573 sexes.

Despite there being no significant sex-differences in task performance, our task-based

575 functional connectivity analysis revealed a more detailed picture of how women's and men's memory

576 performance was related to task-based connectivity across the adult lifespan. Specifically, by using

577 PLS functional connectivity analysis, we were able to consider both within- and between-sex

578 differences in memory accuracy and age in relation to task-based connectivity. In general, using this

579 approach we found three main findings. First, consistent with our prediction, women and men largely

580 showed similar performance-related patterns of functional connectivity. Second, consistent with our

581 prediction, we observed age-related decreases in within-network connectivity and increases in between-

582 network connectivity that was also related to poorer memory performance in advanced age; however,

583 counter to our prediction, this was only observed in men. Women showed age-related decreases in

584 functional connectivity associated with worse memory performance. Below, we detail our PLS

585 connectivity findings and further describe how they complement our previous investigations of brain-

586 behaviour relations in functional activity in a similar cohort.

588 Sex similarities and differences in the effect of accuracy on memory-related brain connectivity

Consistent with our behavioural findings indicating no significant sex differences in memory

performance, we observed similarities in performance-related functional connectivity patterns in

592 women and men in LV2 and LV3. LV2 indicated that during episodic encoding and retrieval, increased

593 between-network connectivity of DMN, FPN and DAN networks with CON, Auditory and SSM

594 networks, respectively, was associated with poorer memory performance for both SE and SH tasks in

595 men, and poorer memory accuracy for SE tasks in women. LV2 also indicated that poorer performance

596 in both sexes was associated with no connectivity of Visual and VAN with other networks. LV3 
597 indicated that greater connectivity within visual, VAN and Salience networks, and between visual-

598 VAN and Salience-FPN networks were related to poorer memory performance for the SE tasks in men 599 and across both task conditions in women. In contrast, the negative correlations from LV3 (Figure 4D) 600 indicates that greater connectivity within DAN, and between DAN-FPN and Salience, Visual-FPN and 601 Salience, VAN-FPN, was correlated with better task performance for SE task in men and both tasks in 602 women. However, these negative correlations were sparse compared to the positive correlations 603 observed in LV3.

604 Previous task-based functional connectivity analysis of visual episodic memory tasks has 605 indicated that greater connectivity between higher-order cognitive networks, i.e., FPN, DMN and the 606 DAN, with task-relevant visual processing networks, i.e., Visual and VAN, is associated with 607 recollection (Bowen \& Kensinger, 2017; King, Chastelaine, Elward, Wang, \& Rugg, 2015). In 608 contrast, Sneve et al. (2017) found that greater intrinsic connectivity between task-irrelevant perceptual 609 networks and cognitive networks during rest, was associated with poorer performance on an associative 610 encoding task performed outside of the scanner. In the current visual spatial context memory study, we 611 found that between-network connectivity of cognitive networks, i.e., DMN, DAN and FPN, with CON 612 and task-irrelevant sensory networks, i.e., Auditory and SSM, during encoding and retrieval was 613 associated with poorer memory performance across all tasks for men, and SE tasks for women. Poorer 614 memory performance was also associated with weak/absent connections between these cognitive 615 networks and Visual and VAN (LV2), and greater connectivity within and between Visual and VAN 616 (LV3). Interestingly, connectivity between the CON, a network hypothesized to be critical for 617 establishing and maintaining task-related performance demands (Dosenbach et al., 2006), with other 618 cognitive networks, i.e., FPN, was not associated with better task performance. This is contrary to prior 619 results highlighting the importance of CON connectivity with other cognitive networks, particularly the 620 FPN, for support for cognitive task performance (Shen et al., 2018). One possibility is that when CON621 cognitive network connectivity is paralleled by increased connectivity between these same cognitive 
networks and task-irrelevant sensory networks, performance is negatively impacted (LV2). Therefore,

our results support the hypothesis that successful associative encoding and retrieval in women and men was correlated with the degree to which higher order cognitive networks were functionally connected with task-irrelevant, compared to task-relevant, sensory networks, and highlight the importance of neural context in determining whether between-network integration of $\mathrm{CON}$ with other higher order cognitive networks was beneficial to task performance or not (McIntosh, 2004).

Prior studies of visual episodic memory tasks have also indicated that FPN connectivity with

DAN supports episodic memory retrieval (Benoit \& Schacter, 2015; Cabeza \& St Jacques, 2007; Kim, also found that memory performance in women and men was differentially correlated to distinct patterns of Salience network connectivity in LV3. It has been hypothesized that connectivity between Salience network and FPN supports externally oriented task performance (Uddin, 2015). Here we 635 found that successful memory performance was associated with Salience network connectivity with DAN; and with VAN and Visual network connectivity with FPN and Salience, respectively. However, the loading and density matrices for LV3 negative correlations indicate the negative correlations were sparse, suggesting segregated patterns of connections involving FPN and Salience with visual systems were important for successful memory performance.

Although we observed significant evidence of similarities in performance-based connectivity in

641 women and men, we also observed some sex differences (LV1). We found that between-network 642 connectivity of the Visual network with DAN, CON, FPN, Salience and SSM during encoding and 643 retrieval was associated with better memory performance during SH tasks in women, and poorer 644 memory performance across all tasks in men. Interestingly, LV3 had a similar pattern of negative 645 correlations between Visual and the aforementioned brain networks and was associated with better 646 memory performance on all tasks in women, and better performance on SE tasks in men. But, in LV3 
647 the Visual network was not densely connected to DAN. Moreover, LV3 indicated Visual-VAN

648 connectivity was negatively correlated with performance in both sexes. The VAN responds to

649 unexpected stimuli (Corbetta and Shulman, 2002; Macaluso et al., 2002; Vossel et al., 2006; 2009),

650 while the DAN regulates perceptual attention for goal-directed task completion and is more relevant to

651 the current task demands (Dixon et al., 2018). Thus, in women, spatial context memory task

652 performance was differentially related to the specific attentional network the Visual network was

653 functionally connected to during encoding and retrieval; with Visual network connectivity to DAN and

654 other higher cognitive networks being associated with better during the more challenging SH task. This

655 pattern of task-based connectivity in women is generally consistent with our previous study looking at

656 fMRI activation, which showed that dorsolateral prefrontal and parietal activation during encoding was

657 correlated with better subsequent performance for SH tasks in women (Subramaniapillai et al., 2019).

658 Importantly, although women did not directly recruit FPN networks, they did recruit the DAN, which is 659 connected with the FPN to regulate perceptual attention for successful task completion (Dixon et al., 660 2018). Therefore, our results indicate that Visual network integration with other cognitive networks 661 was more relevant to memory performance in women compared to men.

Sex differences in the effect of age on memory-related connectivity

Although successful memory performance was correlated with similar patterns of brain

665 connectivity during encoding and retrieval in women and men, there were sex differences in the effect

666 of age on memory-related functional connectivity. This suggests that age-related spatial context

667 memory decline was associated with altered task-based connectivity in distinct brain networks in

668 women, compared to men. In women, spatial context memory performance across all tasks was

669 correlated with greater integration of the Visual network with other cognitive networks (LV1 and LV3)

670 and DAN-FPN connectivity (LV3), and performance on the more difficult SH tasks was related to

671 greater integration of the Visual network with DAN and other cognitive networks (LV1). With age, 
672 women generally showed age-related decreases in these patterns of connectivity. Thus, while older

673 women's reduced ability to engage these networks was not associated with worse task performance

674 during the SE task, it did result in worse SH task performance, suggesting a failure to externally direct

675 attention and engage in spatial processing, particularly with increasing task demands (Corbetta, Patel,

676 \& Shulman, 2008; Grigg \& Grady, 2010; Gusnard, Akbudak, Shulman, \& Raichle, 2001; Spreng,

677 Stevens, Chamberlain, Gilmore, \& Schacter, 2010). In sum, worse memory performance in older

678 women was associated with their inability to successfully integrate task-relevant sensory and cognitive

679 networks to support their performance.

680 In men, spatial context memory performance was positively correlated with decreased

681 functional connectivity between task-irrelevant Auditory and SSM networks (LV2) and task-relevant

682 Visual network (LV1) with higher order cognitive networks (i.e., CON, DMN, DAN, FPN). However,

683 increased age was correlated with greater connectivity between task-irrelevant sensory networks and

684 higher cognitive networks (LV2). Age-related increases in between-network connectivity (integration)

685 has been reported in prior fMRI studies in which biological sex and gender was not used to

686 disaggregate connectivity analyses (Chan et al., 2014; Geerligs et al., 2015; King et al., 2018; Onoda et

687 al., 2012). These age-related differences in functional connectivity are generally associated with age-

688 related cognitive decline and are consistent with theories of age-related neural dedifferentiation that

689 propose neural representations of specific cognitive functions become less distinct with increased age.

690 In the current study, by disaggregating our data by biological sex, we found that these age-related

691 patterns of increased between-network connectivity were specific to men. However, it is notable, that

692 in men advanced age was also associated with reduced within-network connectivity (Salience, VAN,

693 Visual), increased within-network connectivity of DAN, and greater between-network connectivity of

694 DAN-FPN and Salience, which was associated with better performance on the spatial context task at

695 lower task demands (i.e., SE tasks in LV3). This highlights how age-related segregation of brain

696 networks may be modulated by task difficulty. 
698 findings (Subramaniapillai et al., 2019). Both studies interrogate sex differences with age and memory accuracy at different levels of scale in the brain (i.e., regional- vs network-level) using different analyses (activity vs. connectivity), so findings may not completely corroborate with each other. For example, our previous study found age-related deficits in spatial context memory that were primarily associated with altered brain activity at encoding in both women and men, although the pattern of activation differed in both sexes. Interestingly, in our prior activation analyses of these data

704 (Subramaniapillai et al., 2019) and another fMRI study of spatial context memory in older adults at risk of Alzheimer's Disease, we observed greater activation in women, compared to men, in task-relevant sensory networks and higher-order cognitive brain regions that supported memory function (Rabipour et al., 2021). We interpreted these results as potentially reflecting age-related generalization of cognitive function. The current findings indicate that patterns observed in activation and connectivity analyses may be complementary in the sexes. Such that, activation and connectivity increase in taskrelevant sensory networks with cognitive networks benefit memory performance in women, and agerelated decreases in memory function in women is related to decreases in these patterns of connectivity but complementary increases in activation - perhaps to compensate for connectivity deficits. In contrast, in our prior work we did not observed strong patterns of age-related increases in activation in

714 men, but we did see age-related decreases in brain activation. Successful performance was related to

715 distinct/segregated and task-specific patterns of activation. Here, we see that men exhibited age-related 716 increases in between-network integration of task-irrelevant sensory networks with control networks and 717 this was associated with age-related decreases in memory performance. Therefore, in men we observed more generalized patterns of connectivity but not activation with age. In sum, the nature of our current investigation enables an overview of whole brain functional networks on a much broader level in both sexes, while our previous functional activation studies permitted more region-specific understanding of sex differences in age and brain activity, and how these differences directly relate to performance. 
722

723

724

725

726

727

728

729

730

731

732

733

734

735

736

737

738

739

740

741

742

743

744

745

746

\section{Caveats}

The present study examined sex similarities and differences in episodic/context memory across the lifespan using a novel functional connectivity methodological approach. However, our study has several limitations that future work should address. First and foremost, a comprehensive data collection approach was not used when collecting participants' biological sex. Our current study acquired participants' biological sex through self-report, although it could also be ascertained through other means, including participants' sex hormone measurements. Hormone collection is especially relevant when investigating major life transitions, such as menopause, which is associated with age-related differences in women's hormonal profiles. As a consequence of women's greater menopause-related hormonal changes and the established literature of memory effects during this transition (Henderson, 2010; Li et al., 2014; Rentz et al., 2017; Yonker et al., 2006), we decided to omit our cohort of women transitioning through menopause and those who underwent hormone replacement therapy. Although our small cohort size of women in the menopause transition prevented us from including them in our primary analysis, it is essential that future research integrate important life transitions to better inform our understanding of healthy aging models in women and men. Lastly, given that we did not collect information about participants' sociocultural gender, it is further challenging to disentangle the effects of biological sex and sociocultural gender on age- and memory-related connectivity differences.

Our relatively small cohort size constituted another limitation of the current study. Despite the small cohort, our findings complement our previous activation studies, both at the behavioural and functional level, using the same lifespan cohort (Subramaniapillai et al., 2019) and including the larger cohort (n>100; Ankudowich et al., 2016; 2017). Moreover, we found that our PLS connectivity findings were robust to several methodological confounds. First, one challenge that we foresaw was that sex differences in intracranial volume (ICV), with men typically having greater ICV than women, may be driving our functional connectivity results. However, when we ran our analysis on a smaller cohort of participants matched on ICV (and age and education), our findings corroborate our primary 
analysis (Supplementary Figure 2). However, we recognize that this supplementary analysis was conducted on a subcohort of participants with a smaller sample size. Although our primary analysis used a subcohort of participants from the full lifespan cohort, our sample was limited by the smaller cohort of male participants. Strategies are needed to motivate male participants, particularly middleaged men, to participate in research studies. However, we addressed our concerns of not integrating the our primary analysis.

resulted in the same interpretations of findings, whether or not we chose to regress mean task-related

activity or not. Future work should endeavour to understand what these minute differences mean at the node level, both theoretically and conceptually. Thus, although there was the possibility of several confounds, our supplementary analyses findings demonstrate our primary analysis was robust to different preprocessing strategies and methodological confounds.

\section{Conclusions}

Our results highlight similarities in performance-related patterns of task-based connectivity impacts memory-related brain connectivity. Aging was correlated with increased between-network 
772 between task-relevant Visual network and higher order cognitive networks, including DAN. This

773 suggests that the neural basis of age-related episodic memory decline differs in women and men.

774 Evidently, task-based connectivity and resting state connectivity both have an important and

775 complementary role in studying sex differences in healthy aging and cognition. Our findings further

776 cement the importance of separately understand healthy aging models for women and men to

777 understand how healthy aging that eventually lead to neurodegenerative disorders, such as Alzheimer's

778 or Parkinson's, that differentially impact women and men.

779

780 Acknowledgements: This work was supported by CIHR Operating Grants (GS9-171369

781

and 201610PJT) and NSERC Discovery Grant (RGPIN-2018-05761) awarded to M.N. Rajah; Canada

782 Research Chair II; the Natural Science and Engineering Research Council Graham Bell Canada

783 Graduate Scholarship-Doctoral and the Healthy Brains Healthy Lives Doctoral Fellowship awarded to

784 S. Subramaniapillai.

785

786

Contributions: M. N. Rajah (MNR) designed the study. S. Subramaniapillai (SS), S. Rajagopal (SR)

and E. Ankudowich (EA) contributed to data processing and analysis. Stamatoula Pasvanis (SP) and

EA led data collection and quality control. SS and SR created figures and tables. Bratislav Misic (BM)

provided the PLS connectivity code, SR edited and created the GitHub code used in the current

publication. SS, SR, EA, BM, and M. N. Rajah (MNR) provided analytic, theoretical input and in results interpretation and writing of introduction and discussion. 
797

798

799

800

801

802

803

804

805

806

807

808

809

810

811

812

813

814

815

816

817

818

819

820

821

\section{References}

2020 Alzheimer's disease facts and figures. (2020). Alzheimer's and Dementia, 16(3), 391-460. https://doi.org/10.1002/alz.12068

Adnan, A., Beaty, R., Lam, J., Spreng, R. N., \& Turner, G. R. (2019). Intrinsic default-executive coupling of the creative aging brain. Social Cognitive and Affective Neuroscience, 14(3), 291-303. https://doi.org/10.1093/scan/nsz013

Allen, E. A., Erhardt, E. B., Damaraju, E., Gruner, W., Segall, J. M., Silva, R. F., ... Calhoun, V. D. (2011). A baseline for the multivariate comparison of resting-state networks. Frontiers in Systems Neuroscience, 5(FEBRUARY 2011), 1-23. https://doi.org/10.3389/fnsys.2011.00002

Amer, T., Anderson, J. A. E., Campbell, K. L., Hasher, L., \& Grady, C. L. (2016). Age differences in the neural correlates of distraction regulation: A network interaction approach. NeuroImage, 139, 231-239. https://doi.org/10.1016/j.neuroimage.2016.06.036

Amer, T., Campbell, K. L., \& Hasher, L. (2016). Cognitive Control As a Double-Edged Sword. Trends in Cognitive Sciences, 20(12), 905-915. https://doi.org/http://dx.doi.org/10.1016/j.tics.2016.10.002

Andrews-Hanna, J. R., Smallwood, J., \& Spreng, N. (2014). The default network and self-generated thought: component processes,dynamic control, and clinical relevance. Psychology and Aging, 1316(1), 29-52. https://doi.org/. doi:10.1111/nyas.12360

Ankudowich, E., Pasvanis, S., \& Rajah, M. N. (2016). Changes in the modulation of brain activity during context encoding vs. context retrieval across the adult lifespan. NeuroImage, 139, 103-113. https://doi.org/10.1016/j.neuroimage.2016.06.022

Ankudowich, E., Pasvanis, S., \& Rajah, M. N. (2017). Changes in the correlation between spatial and temporal source memory performance and BOLD activity across the adult lifespan. Cortex, 91, 234-249. https://doi.org/10.1016/j.cortex.2017.01.006

Antonenko, D., \& Flöel, A. (2013). Healthy aging by staying selectively connected: A mini-review. 
Asperholm, M., Van Leuven, L., \& Herlitz, A. (2020). Sex differences in episodic memory variance.

Astur, R. S., Purton, A. J., Zaniewski, M. J., Cimadevilla, J., \& Markus, E. J. (2016). Human sex differences in solving a virtual navigation problem. Behavioural Brain Research, 308, 236-243. https://doi.org/10.1016/j.bbr.2016.04.037

Avelar-Pereira, B., Bäckman, L., Wåhlin, A., Nyberg, L., \& Salami, A. (2017). Age-related differences in dynamic interactions among default mode, frontoparietal control, and dorsal attention networks during resting-state and interference resolution. Frontiers in Aging Neuroscience, 9(MAY), 1-15. https://doi.org/10.3389/fnagi.2017.00152

Bates, D., Mächler, M., Bolker, B. M., \& Walker, S. C. (2015). Fitting linear mixed-effects models using lme4. Journal of Statistical Software, 67(1). https://doi.org/10.18637/jss.v067.i01

Betzel, R. F., Byrge, L., He, Y., Goñi, J., Zuo, X. N., \& Sporns, O. (2014). Changes in structural and

Birn, R. M., Cornejo, M. D. aniel., Molloy, E. K., Patriat, R., Meier, T. B., Kirk, G. R., ... Prabhakaran, V. (2014). The influence of physiological noise correction on test-retest reliability of

Biswal, B. B., Mennes, M., Zuo, X. N., Gohel, S., Kelly, C., Smith, S. M., .. Milham, M. P. (2010). Toward discovery science of human brain function. Proceedings of the National Academy of Sciences of the United States of America, 107(10), 4734-4739. https://doi.org/10.1073/pnas.0911855107

Biswal, B., Zerrin Yetkin, F., Haughton, V. M., \& Hyde, J. S. (1995). Functional connectivity in the motor cortex of resting human brain using echo-planar mri. Magnetic Resonance in Medicine, 
848 Bluhm, R. L., Osuch, E. A., Lanius, R. A., Boksman, K., Neufeld, R. W. J., Théberge, J., \& Williamson, P. (2008). Default mode network connectivity: Effects of age, sex, and analytic approach. NeuroReport, 19(8), 887-891. https://doi.org/10.1097/WNR.0b013e328300ebbf

Bowen, H. J., \& Kensinger, E. A. (2017). Memory-related functional connectivity in visual processing regions varies by prior emotional context. NeuroReport, 28(13), 808-813. https://doi.org/10.1097/WNR.0000000000000829 Psychopharmacology Bulletin, 35(3), 55-78. Retrieved from http://europepmc.org/abstract/MED/12397879

Bullmore, E., \& Sporns, O. (2012). The economy of brain network organization. Nature Reviews

860 Cao, M., Wang, J. H., Dai, Z. J., Cao, X. Y., Jiang, L. L., Fan, F. M., ... He, Y. (2014). Topological organization of the human brain functional connectome across the lifespan. Developmental

Cerri, S., Mus, L., \& Blandini, F. (2019). Parkinson's Disease in Women and Men: What's the Difference? Journal of Parkinson's Disease, 9(3), 501-515. https://doi.org/10.3233/JPD-191683 
872 Corbetta, M., Patel, G., \& Shulman, G. L. (2008). The Reorienting System of the Human Brain: From 873 Environment to Theory of Mind. 2Neuron, 58(3), 306-324.

874 Damoiseaux, J. S., Beckmann, C. F., Arigita, E. J. S., Barkhof, F., Scheltens, P., Stam, C. J., ...

Rombouts, S. A. R. B. (2008). Reduced resting-state brain activity in the "default network" in normal aging. Cerebral Cortex, 18(8), 1856-1864. https://doi.org/10.1093/cercor/bhm207

Damoiseaux, Jessica S. (2017). Effects of aging on functional and structural brain connectivity. NeuroImage, 160(January), 32-40. https://doi.org/10.1016/j.neuroimage.2017.01.077 Evidence from Functional Brain Connectivity and Spinal Fluid Tau Levels. JOURNAL OF NEUROSCIENCE, 32(24), 8254-8262. https://doi.org/10.1523/JNEUROSCI.0305-12.2012

Dixon, M. L., Andrews-Hanna, J. R., Spreng, R. N., Irving, Z. C., Mills, C., Girn, M., \& Christoff, K. (2017). Interactions between the default network and dorsal attention network vary across default subsystems, time, and cognitive states. NeuroImage, 147(July 2016), 632-649. https://doi.org/10.1016/j.neuroimage.2016.12.073

Dixon, M. L., De La Vega, A., Mills, C., Andrews-Hanna, J., Spreng, R. N., Cole, M. W., \& Christoff, K. (2018). Heterogeneity within the frontoparietal control network and its relationship to the default and dorsal attention networks. Proceedings of the National Academy of Sciences, 115(7), E1598-E1607. https://doi.org/10.1073/pnas.1715766115 25(8), 798-806. https://doi.org/10.1002/gps.2420

894 Dosenbach, N. U. F., Visscher, K. M., Palmer, E. D., Miezin, F. M., Wenger, K. K., Kang, H. C., ... 
897 Esposito, R., Cieri, F., Chiacchiaretta, P., Cera, N., Lauriola, M., Di Giannantonio, M., ... Ferretti, A.

898 (2018). Modifications in resting state functional anticorrelation between default mode network and 899 dorsal attention network: comparison among young adults, healthy elders and mild cognitive impairment patients. Brain Imaging and Behavior, 12(1), 127-141.

901 https://doi.org/10.1007/s11682-017-9686-y

902

Ferreira, L. K., Regina, A. C. B., Kovacevic, N., Martin, M. D. G. M., Santos, P. P., Carneiro, C. D. G., 903 ... Busatto, G. F. (2016). Aging effects on whole-brain functional connectivity in adults free of cognitive and psychiatric disorders. Cerebral Cortex, 26(9), 3851-3865.

905 https://doi.org/10.1093/cercor/bhv190

Filippi, M., Valsasina, P., Misci, P., Falini, A., Comi, G., \& Rocca, M. A. (2013). The organization of intrinsic brain activity differs between genders: A resting-state fMRI study in a large cohort of young healthy subjects. Human Brain Mapping, 34(6), 1330-1343. https://doi.org/10.1002/hbm.21514

910 Fox, M. D., Snyder, A. Z., Vincent, J. L., Corbetta, M., Van Essen, D. C., \& Raichle, M. E. (2005). The human brain is intrinsically organized into dynamic, anticorrelated functional networks. Proceedings of the National Academy of Sciences of the United States of America, 102(27), 96739678. https://doi.org/10.1073/pnas.0504136102

Friston, K. J. (1994). Functional and effective connectivity in neuroimaging: A synthesis. Human Brain Mapping, 2(1-2), 56-78. https://doi.org/10.1002/hbm.460020107

Geerligs, L., Renken, R. J., Saliasi, E., Maurits, N. M., \& Lorist, M. M. (2015). A Brain-Wide Study of

919 Grady, C. L., \& Craik, F. I. (2000). Changes in memory processing with age. Current Opinion in 920 Neurobiology, 10(2), 224-231. https://doi.org/10.1016/S0959-4388(00)00073-8

921 Grady, C. L., Protzner, A. B., Kovacevic, N., Strother, S. C., Afshin-Pour, B., Wojtowicz, M., ... 
McIntosh, A. R. (2010). A multivariate analysis of age-related differences in default mode and task-positive networks across multiple cognitive domains. Cerebral Cortex, 20(6), 1432-1447. https://doi.org/10.1093/cercor/bhp207

Grady, C., Sarraf, S., Saverino, C., \& Campbell, K. (2016). Age differences in the functional interactions among the default, frontoparietal control, and dorsal attention networks. Neurobiology of Aging, 41, 159-172. https://doi.org/10.1016/j.neurobiolaging.2016.02.020

Grigg, O., \& Grady, C. L. (2010). The default network and processing of personally relevant information: Converging evidence from task-related modulations and functional connectivity. Neuropsychologia, 48(13), 3815-3823. https://doi.org/10.1016/j.neuropsychologia.2010.09.007

Gusnard, D. A., Akbudak, E., Shulman, G. L., \& Raichle, M. E. (2001). Medial prefrontal cortex and self-referential mental activity: Relation to a default mode of brain function. Proceedings of the National Academy of Sciences of the United States of America, 98(7), 4259-4264. https://doi.org/10.1073/pnas.071043098

Hayama, H. R., Vilberg, K. L., \& Rugg, M. D. (2012). Overlap between the neural correlates of cued recall and source memory: Evidence for a generic recollection network? Journal of Cognitive Neuroscience, 24(5), 1127-1137.https://doi.org/10.1162/jocn_a_00202

He, J., Carmichael, O., Fletcher, E., Singh, B., Iosif, A.-M., Martinez, O., ... DeCarli, C. (2012). Influence of functional connectivity and structural MRI measures on episodic memory. Neurobiology of Aging, 33(11), 2612-2620. Retrieved from \%3CGo

Henderson, V. W. (2010). Action of estrogens in the aging brain: Dementia and cognitive aging. Biochimica et Biophysica Acta - General Subjects, 1800(10), 1077-1083. https://doi.org/10.1016/j.bbagen.2009.11.005

Herlitz, a, Nilsson, L. G., \& Bäckman, L. (1997). Gender differences in episodic memory. Memory \& Cognition, 25(6), 801-811. https://doi.org/10.3758/BF03211324

Herlitz, A., \& Rehnman, J. (2008). Sex differences in episodic memory. Current Directions in 
Psychological Science, 17(1), 52-56. https://doi.org/10.1111/j.1467-8721.2008.00547.x

948 Huo, L., Li, R., Wang, P., Zheng, Z., \& Li, J. (2018). The Default Mode Network Supports Episodic Memory in Cognitively Unimpaired Elderly Individuals: Different Contributions to Immediate Recall and Delayed Recall. Frontiers in Aging Neuroscience, 10. Retrieved from \%3CGo

Jing, H. G., Madore, K. P., \& Schacter, D. L. (2020). Not to Worry: Episodic Retrieval Impacts Emotion Regulation in Older Adults. Emotion, 1-31.

Johnson, J. D., \& Rugg, M. D. (2007). Recollection and the reinstatement of encoding-related cortical activity. Cerebral Cortex, 17(11), 2507-2515. https://doi.org/10.1093/cercor/bhl156

Keightley, M. L., Winocur, G., Burianova, H., Hongwanishkul, D., \& Grady, C. L. (2006). Age effects on social cognition: Faces tell a different story. Psychology and Aging, 21(3), 558-572. https://doi.org/10.1037/0882-7974.21.3.558

King, B. R., Van Ruitenbeek, P., Leunissen, I., Cuypers, K., Heise, K. F., Santos Monteiro, T., ... Swinnen, S. P. (2018). Age-related declines in motor performance are associated with decreased segregation of large-scale resting state brain networks. Cerebral Cortex, 28(12), 4390-4402. https://doi.org/10.1093/cercor/bhx297

King, D. R., de Chastelaine, M., \& Rugg, M. D. (2018). Recollection-related increases in functional connectivity across the healthy adult lifespan. NEUROBIOLOGY OF AGING, 62, 1-19. https://doi.org/10.1016/j.neurobiolaging.2017.09.026

King, X. R., Chastelaine, M. De, Elward, R. L., Wang, T. H., \& Rugg, M. D. (2015). RecollectionRelated Increases in Functional Connectivity Predict Individual Differences in Memory Accuracy. Journal of Neuroscience, 35(4), 1763-1772. https://doi.org/10.1523/JNEUROSCI.3219-14.2015

Koller, M. (2016). Robustlmm: An R package for Robust estimation of linear Mixed-Effects models. Journal of Statistical Software, 75(1). https://doi.org/10.18637/jss.v075.i06

Kwon, D., Maillet, D., Pasvanis, S., Ankudowich, E., Grady, C. L., \& Rajah, M. N. (2016). Context Memory Decline in Middle Aged Adults is Related to Changes in Prefrontal Cortex Function. 
Cerebral Cortex , 26(6), 2440-2460. https://doi.org/10.1093/cercor/bhv068

973 Lewin, C., Wolgers, G., \& Herlitz, A. (2001). Sex differences favoring women in verbal but not in 974 visuospatial episodic memory. Neuropsychology, 15(2), 165-173. https://doi.org/10.1037/08944105.15.2.165

Li, R., Cui, J., \& Shen, Y. (2014). Brain sex matters: Estrogen in cognition and Alzheimer's disease. Molecular and Cellular Endocrinology, 389(1-2), 13-21. https://doi.org/10.1016/j.mce.2013.12.018

Li, S. C., Lindenberger, U., \& Sikström, S. (2001). Aging cognition: From neuromodulation to representation. Trends in Cognitive Sciences, 5(11), 479-486. https://doi.org/10.1016/S13646613(00)01769-1

Mano, Y., Sugiura, M., Tsukiura, T., Chiao, J. Y., Yomogida, Y., Jeong, H., ... Kawashima, R. (2011). The representation of social interaction in episodic memory: A functional MRI study. NeuroImage, 57(3), 1234-1242. https://doi.org/10.1016/j.neuroimage.2011.05.016

McIntosh, Anthony R. (2000). From location to integration: How neural interactions form the basis for human cognition. Memory, Consciousness and the Brain: The Tallin Conference, 346-362.

McIntosh, Anthony Randal. (2004). Contexts and catalysts: A resolution of the localization and

Mesulam, M. (1990). Large-Scale neurocognitive networks and distributed processing for attention, language, and memory. Neurological Progress, 28, 597-613.

Mevel, K., Landeau, B., Fouquet, M., La Joie, R., Villain, N., Mézenge, F., ... Chételat, G. (2013). Age 
998 Mohr, H., Wolfensteller, U., Betzel, R. F., Mišić, B., Sporns, O., Richiardi, J., \& Ruge, H. (2016). Integration and segregation of large-scale brain networks during short-term task automatization. Nature Communications, 7. https://doi.org/10.1038/ncomms13217

Mol, M., Carpay, M., Ramakers, I., Rozendaal, N., Verhey, F., \& Jolles, J. (2007). The effect of percieved forgetfulness on quality of life in older adults; a qualitative review. International Journal of Geriatric Psychiatry, 22(5), 393-400. https://doi.org/10.1002/gps.1686

Nathan Spreng, R., Dupre, E., Selarka, D., Garcia, J., Gojkovic, S., Mildner, J., ... Turner, G. R. (2014). Goal-congruent default network activity facilitates cognitive control. Journal of Neuroscience, 34(42), 14108-14114. https://doi.org/10.1523/JNEUROSCI.2815-14.2014

Ng, K. K., Lo, J. C., Lim, J. K. W., Chee, M. W. L., \& Zhou, J. (2016). Reduced functional segregation between the default mode network and the executive control network in healthy older adults: A longitudinal study. NeuroImage, 133, 321-330. https://doi.org/10.1016/j.neuroimage.2016.03.029

1017 Onoda, K., Ishihara, M., \& Yamaguchi, S. (2012). Decreased functional connectivity by aging is associated with cognitive decline. Journal of Cognitive Neuroscience, 24(11), 2186-2198. https://doi.org/10.1162/jocn_a_00269 

https://doi.org/10.1523/JNEUROSCI.4494-11.2012

Power, J. D., Cohen, A. L., Nelson, S. M., Wig, G. S., Barnes, K. A., Church, J. A., .. Petersen, S. E. (2011). Functional Network Organization of the Human Brain. Neuron, 72(4), 665-678.

Prakash, R. S., Heo, S., Voss, M. W., Patterson, B., \& Kramer, A. F. (2012). Age-related differences in cortical recruitment and suppression: Implications for cognitive performance. Behavioural Brain Research, 230(1), 192-200. https://doi.org/10.1016/j.bbr.2012.01.058

Rabipour, S., Rajagopal, S., Pasvanis, S., \& Rajah, M. N. (2021). Generalization of memory-related brain function in asymptomatic older women with a family history of late onset Alzheimer's Disease: Results from the PREVENT-AD Cohort. Neurobiology of Aging, 104, 42-56. https://doi.org/10.1016/j.neurobiolaging.2021.03.009 cortex activity during spatial and temporal context memory retrieval after equating for task

Ragland, J. D., Coleman, A. R., Gur, R. C., Glahn, D. C., \& Gur, R. E. (2000). Sex differences in brain-

Rajah, M. N., Languay, R., \& Valiquette, L. (2010). Age-related changes in prefrontal cortex activity are associated with behavioural deficits in both temporal and spatial context memory retrieval in older adults. Cortex, 46(4), 535-549. https://doi.org/10.1016/j.cortex.2009.07.006

1044 Rajah, M. N., McIntosh, A. R., \& Grady, C. L. (1999). Frontotemporal interactions in face encoding and recognition. Cognitive Brain Research, 8(3), 259-269. https://doi.org/10.1016/S0926- 
1047 Rentz, D. M., Weiss, B. K., Jacobs, E. G., Cherkerzian, S., Klibanski, A., Remington, A., ... Goldstein, 1048 J. M. (2017). Sex differences in episodic memory in early midlife: Impact of reproductive aging. Menopause, 24(4), 400-408. https://doi.org/10.1097/GME.0000000000000771

1050 Rieck, J. R., Rodrigue, K. M., Boylan, M. A., \& Kennedy, K. M. (2017). Age-related reduction of BOLD modulation to cognitive difficulty predicts poorer task accuracy and poorer fluid reasoning ability. NeuroImage, 147(July 2016), 262-271. https://doi.org/10.1016/j.neuroimage.2016.12.022

Rugg, M. D. (2017). Interpreting age-related differences in memory-related neural activity. In Cognitive neuroscience of aging: Linking cognitive and cerebral aging, 2nd ed. (pp. 183-203). New York, NY, US: Oxford University Press.

Sala-Llonch, R., Peña-Gómez, C., Arenaza-Urquijo, E. M., Vidal-Piñeiro, D., Bargalló, N., Junqué, C., \& Bartrés-Faz, D. (2012). Brain connectivity during resting state and subsequent working memory task predicts behavioural performance. Cortex, 48(9), 1187-1196. https://doi.org/10.1016/j.neuron.2012.11.001.The

Scheinost, D., Finn, E. S., Tokoglu, F., Shen, X., Papademetris, X., Hampson, M., \& Constable, R. T. (2015). Sex differences in normal age trajectories of functional brain networks. Human Brain Mapping, 36(4), 1524-1535. https://doi.org/10.1002/hbm.22720 (2018). Resting-State Connectivity and Its Association With Cognitive Performance, Educational Attainment, and Household Income in the UK Biobank. Biological Psychiatry: Cognitive Neuroscience and Neuroimaging, 3(10), 878-886. https://doi.org/10.1016/j.bpsc.2018.06.007 Siman-Tov, T., Bosak, N., Sprecher, E., Paz, R., Eran, A., Aharon-Peretz, J., \& Kahn, I. (2017). Early age-related functional connectivity decline in high-order cognitive networks. Frontiers in Aging 
1073 Spaniol, J., Davidson, P. S. R., Kim, A. S. N., Han, H., Moscovitch, M., \& Grady, C. L. (2009). Eventrelated fMRI studies of episodic encoding and retrieval: Meta-analyses using activation likelihood estimation. Neuropsychologia, 47(8-9), 1765-1779. https://doi.org/10.1016/j.neuropsychologia.2009.02.028

Spencer, W. D., \& Raz, N. (1995). Differential effects of aging on memory for context and content: A meta-analysis. Psychology and Aging, 10(4), 527-539.

Sporns, O. (2013). Network attributes for segregation and integration in the human brain. Current Opinion in Neurobiology, 23(2), 162-171. https://doi.org/10.1016/j.conb.2012.11.015 640. https://doi.org/10.1146/annurev-psych-122414-033634

Spreng, N., Stevens, D., Chamberlain, J. P., Gilmore, A., \& Schacter, D. (2010). Default network activity, coupled with the frontoparietal control network, network, supports goal-directed cognition. Neuroimage, 53(1), 303-317.

Spreng, R. N., Lockrow, A. W., DuPre, E., Setton, R., Spreng, K. A. P., \& Turner, G. R. (2018). Semanticized autobiographical memory and the default - executive coupling hypothesis of aging. Neuropsychologia, 110(May 2017), 37-43.

Spreng, R. N., Stevens, W. D., Viviano, J. D., \& Schacter, D. L. (2016). Attenuated anticorrelation between the default and dorsal attention networks with aging: evidence from task and rest. pathological aging. Journal of Neuroscience, 33(38), 15226-15234. https://doi.org/10.1523/JNEUROSCI.2261-13.2013

1096 St-Laurent, M., Abdi, H., BurianovìÁ, H., \& Grady, C. L. (2011). Influence of aging on The neural 
correlates of autobiographical, Episodic, and semantic Memory retrieval. Retrieved from -

1098 Strother, S. C., Kanno, I., Rottenberg, D. A., Friston, K. J., \& Ford, I. (1995). Commentary and opinion: I. Principal component analysis, variance partitioning, and "functional connectivity." Journal of Cerebral Blood Flow and Metabolism, 15(3), 353-377.

\section{2} https://doi.org/10.1038/jcbfm.1995.44

Stumme, J., Jockwitz, C., Hoffstaedter, F., Amunts, K., \& Caspers, S. (2020). Functional network reorganization in older adults: Graph-theoretical analyses of age, cognition and sex. NeuroImage,

Team, R. C. (n.d.). A Language and Environment for Statistical Computing. $R$ Core Team. R: A Language and Environment for Statistical Computing. (R Foundation for Statistical Computing, 214(September 2019), 116756. https://doi.org/10.1016/j.neuroimage.2020.116756

Subramaniapillai, S., Almey, A., Natasha Rajah, M., \& Einstein, G. (2021). Sex and gender differences in cognitive and brain reserve: Implications for Alzheimer's disease in women. Frontiers in Neuroendocrinology, 60(February). https://doi.org/10.1016/j.yfrne.2020.100879

Subramaniapillai, S., Rajagopal, S., Elshiekh, A., Pasvanis, S., Ankudowich, E., \& Rajah, M. N. (2019). Sex Differences in the Neural Correlates of Spatial Context Memory Decline in Healthy Aging. Journal of Cognitive Neuroscience, 31(12), 1895-1916. Vienna, Austria, 2013).

Thomas Yeo, B. T., Krienen, F. M., Sepulcre, J., Sabuncu, M. R., Lashkari, D., Hollinshead, M., ... Buckner, R. L. (2011). The organization of the human cerebral cortex estimated by intrinsic functional connectivity. Journal of Neurophysiology, 106(3), 1125-1165. https://doi.org/10.1152/jn.00338.2011

1121 Tomasi, D., \& Volkow, N. D. (2012). Gender differences in brain functional connectivity density. 
1123 Tulving, E. (2002). Episodic Memory: From Mind to Brain. Annu. Rev. Pscyhol., 53, 1-25.

1124 Turner, G. R., \& Spreng, N. (2015). Prefrontal Engagement and Reduced Default Network Suppression

1125 Co-occur and Are Dynamically Coupled in Older Adults: The Default-Executive Coupling

1126 Hypothesis of Aging. Journal of Cognitive Neuroscience, 12, 2462-2476.

1127 Uddin, L. Q. (2015). Salience processing and insular cortical function and dysfunction. Nature Reviews $1128 \quad$ Neuroscience, 16(1), 55-61. https://doi.org/10.1038/nrn3857

1129 Uddin, L. Q., Yeo, B. T. T., \& Spreng, R. N. (2019). Towards a Universal Taxonomy of Macro-scale

1130 Functional Human Brain Networks. Brain Topography, 32(6), 926-942.

$1131 \quad$ https://doi.org/10.1007/s10548-019-00744-6

1132 Varangis, E., Habeck, C. G., \& Stern, Y. (2021). Task-based functional connectivity in aging: How

1133 task and connectivity methodology affect discovery of age effects. Brain and Behavior, 11(1), 1-

1134 15. https://doi.org/10.1002/brb3.1954

1135 Weissman-Fogel, I., Moayedi, M., Taylor, K. S., Pope, G., \& Davis, K. D. (2010). Cognitive and 1136 default-mode resting state networks: Do male and female brains "rest" differently? Human Brain 1137 Mapping, 31(11), 1713-1726. https://doi.org/10.1002/hbm.20968

1138 Yonker, J., Adolfsson, R., Eriksson, E., Hellstrand, M., Nilsson, L. G., \& Herlitz, A. (2006). Verified 1139 hormone therapy improves episodic memory performance in healthy postmenopausal women. $1140 \quad$ Aging, Neuropsychology, and Cognition, 13(3-4), 291-307.

$1141 \quad$ https://doi.org/10.1080/138255890968655 
Supplementary Materials

Supplementary Table 1. Power atlas ROI network nodes $(n=216)$ used in the analysis

\begin{tabular}{|c|c|c|c|c|c|}
\hline \multirow[b]{2}{*}{ Network } & \multirow[b]{2}{*}{ Talaraich Daemon Label } & \multirow[b]{2}{*}{ Brodmann_Area } & \multicolumn{3}{|c|}{ MNI Coordinates } \\
\hline & & & $\mathrm{X}$ & $\mathrm{Y}$ & $\mathrm{Z}$ \\
\hline Auditory & Insula & 13 & 32 & -28 & 12 \\
\hline Auditory & Insula & 13 & 64 & -32 & 20 \\
\hline Auditory & Transverse Temporal & 41 & 56 & -16 & 8 \\
\hline Auditory & STG & 42 & -40 & -32 & 16 \\
\hline Auditory & STG & 41 & -60 & -24 & 12 \\
\hline Auditory & STG & 41 & -48 & -28 & 4 \\
\hline Auditory & Insula & 13 & 44 & -24 & 20 \\
\hline Auditory & Insula & 13 & -48 & -36 & 24 \\
\hline Auditory & Insula & 13 & -52 & -20 & 24 \\
\hline Auditory & PreCent & 43 & -56 & -8 & 12 \\
\hline Auditory & PreCent & 43 & 56 & -4 & 12 \\
\hline Auditory & PostCent & 2 & 60 & -16 & 28 \\
\hline Auditory & Insula & 13 & -32 & -28 & 12 \\
\hline $\mathrm{CON}$ & $\mathrm{mFG}$ & 6 & -4 & 4 & 52 \\
\hline $\mathrm{CON}$ & IPL & 40 & 56 & -28 & 32 \\
\hline $\mathrm{CON}$ & SFG & 6 & 20 & -8 & 64 \\
\hline $\mathrm{CON}$ & SFG & 6 & -16 & -4 & 72 \\
\hline $\mathrm{CON}$ & CG & 24 & -12 & -4 & 44 \\
\hline $\mathrm{CON}$ & $\mathrm{LN}$ & Putamen & 36 & 0 & -4 \\
\hline $\mathrm{CON}$ & SFG & 6 & 12 & 0 & 68 \\
\hline $\mathrm{CON}$ & $\mathrm{mFG}$ & 6 & 8 & 8 & 52 \\
\hline $\mathrm{CON}$ & Insula & 13 & -44 & 0 & 8 \\
\hline $\mathrm{CON}$ & Insula & 13 & 48 & 8 & 0 \\
\hline $\mathrm{CON}$ & STG & 22 & -52 & 8 & -4 \\
\hline $\mathrm{CON}$ & CG & 32 & -4 & 16 & 36 \\
\hline $\mathrm{CON}$ & Claustrum & $*$ & 36 & 12 & 0 \\
\hline $\mathrm{DMN}$ & MTG & 19 & -40 & -76 & 24 \\
\hline $\mathrm{DMN}$ & $\mathrm{mFG}$ & 10 & 4 & 68 & -4 \\
\hline $\mathrm{DMN}$ & $\mathrm{mFG}$ & 10 & 8 & 48 & -16 \\
\hline $\mathrm{DMN}$ & PHG & 30 & -12 & -40 & 0 \\
\hline $\mathrm{DMN}$ & $\mathrm{mFG}$ & 10 & -16 & 64 & -8 \\
\hline $\mathrm{DMN}$ & MTG & 19 & -44 & -60 & 20 \\
\hline $\mathrm{DMN}$ & MTG & 39 & 44 & -72 & 28 \\
\hline $\mathrm{DMN}$ & STG & 38 & -44 & 12 & -36 \\
\hline $\mathrm{DMN}$ & STG & 38 & 44 & 16 & -32 \\
\hline $\mathrm{DMN}$ & MTG & 21 & -68 & -24 & -16 \\
\hline $\mathrm{DMN}$ & MTG & 39 & -44 & -64 & 36 \\
\hline $\mathrm{DMN}$ & $\mathrm{PrCu}$ & 19 & -40 & -76 & 44 \\
\hline $\mathrm{DMN}$ & PostCing & 31 & -8 & -56 & 28 \\
\hline
\end{tabular}




\begin{tabular}{|c|c|c|c|c|c|}
\hline DMN & $\mathrm{PrCu}$ & 7 & 4 & -60 & 36 \\
\hline $\mathrm{DMN}$ & PostCing & 30 & -12 & -56 & 16 \\
\hline $\mathrm{DMN}$ & PostCing & 29 & -4 & -48 & 12 \\
\hline $\mathrm{DMN}$ & $\mathrm{CG}$ & 31 & 8 & -48 & 32 \\
\hline $\mathrm{DMN}$ & $\mathrm{PrCu}$ & 31 & 16 & -64 & 24 \\
\hline $\mathrm{DMN}$ & $\mathrm{CG}$ & 31 & -4 & -36 & 44 \\
\hline DMN & PostCing & 30 & 12 & -52 & 16 \\
\hline DMN & STG & 39 & 52 & -60 & 36 \\
\hline $\mathrm{DMN}$ & SFG & 8 & 24 & 32 & 48 \\
\hline $\mathrm{DMN}$ & SFG & 6 & -12 & 40 & 52 \\
\hline $\mathrm{DMN}$ & SFG & 6 & -16 & 28 & 52 \\
\hline $\mathrm{DMN}$ & MFG & 6 & -36 & 20 & 52 \\
\hline DMN & MFG & 8 & 24 & 40 & 40 \\
\hline $\mathrm{DMN}$ & SFG & 8 & 12 & 56 & 40 \\
\hline $\mathrm{DMN}$ & SFG & 8 & -12 & 56 & 40 \\
\hline $\mathrm{DMN}$ & SFG & 8 & -20 & 44 & 40 \\
\hline $\mathrm{DMN}$ & $\mathrm{mFG}$ & 9 & 4 & 56 & 16 \\
\hline $\mathrm{DMN}$ & SFG & 9 & 8 & 64 & 20 \\
\hline $\mathrm{DMN}$ & $\mathrm{mFG}$ & 10 & -8 & 52 & 0 \\
\hline $\mathrm{DMN}$ & $\mathrm{mFG}$ & 10 & 8 & 56 & 4 \\
\hline $\mathrm{DMN}$ & $\mathrm{ACC}$ & 32 & -4 & 44 & -8 \\
\hline $\mathrm{DMN}$ & $\mathrm{ACC}$ & 32 & 8 & 44 & -4 \\
\hline $\mathrm{DMN}$ & $\mathrm{mFG}$ & 9 & -12 & 44 & 8 \\
\hline DMN & $\mathrm{mFG}$ & 8 & -4 & 36 & 36 \\
\hline $\mathrm{DMN}$ & $\mathrm{ACC}$ & 32 & -4 & 40 & 16 \\
\hline $\mathrm{DMN}$ & SFG & 9 & -20 & 64 & 20 \\
\hline $\mathrm{DMN}$ & $\mathrm{mFG}$ & 9 & -8 & 48 & 24 \\
\hline $\mathrm{DMN}$ & ITG & 21 & 64 & -12 & -20 \\
\hline $\mathrm{DMN}$ & MTG & 21 & -56 & -12 & -12 \\
\hline $\mathrm{DMN}$ & MTG & 21 & -56 & -28 & -4 \\
\hline $\mathrm{DMN}$ & MTG & 21 & 64 & -32 & -8 \\
\hline $\mathrm{DMN}$ & MTG & 21 & -68 & -40 & -4 \\
\hline $\mathrm{DMN}$ & SFG & 6 & 12 & 28 & 60 \\
\hline $\mathrm{DMN}$ & $\mathrm{ACC}$ & 32 & 12 & 36 & 20 \\
\hline $\mathrm{DMN}$ & Sub-Gyral & 21 & 52 & -4 & -16 \\
\hline $\mathrm{DMN}$ & PHG & 36 & -28 & -40 & -8 \\
\hline $\mathrm{DMN}$ & PHG & 36 & 28 & -36 & -12 \\
\hline DMN & FFG & 37 & -32 & -40 & -16 \\
\hline $\mathrm{DMN}$ & STG & 38 & 52 & 8 & -28 \\
\hline $\mathrm{DMN}$ & MTG & 21 & -52 & 4 & -28 \\
\hline DMN & STG & 39 & 48 & -52 & 28 \\
\hline $\mathrm{DMN}$ & MTG & 21 & -48 & -44 & 0 \\
\hline $\mathrm{DMN}$ & IFG & 47 & -48 & 32 & -12 \\
\hline $\mathrm{DMN}$ & MFG & 47 & 48 & 36 & -12 \\
\hline
\end{tabular}




\begin{tabular}{|c|c|c|c|c|c|}
\hline $\mathrm{DMN}$ & $\mathrm{CG}$ & 31 & -4 & -36 & 32 \\
\hline $\mathrm{DMN}$ & $\mathrm{PrCu}$ & 7 & -8 & -72 & 40 \\
\hline $\mathrm{DMN}$ & $\mathrm{PrCu}$ & 7 & 12 & -68 & 44 \\
\hline $\mathrm{DMN}$ & $\mathrm{PrCu}$ & 7 & 4 & -48 & 52 \\
\hline $\mathrm{DMN}$ & CG & 23 & 0 & -24 & 32 \\
\hline DAN & $\mathrm{PrCu}$ & 7 & 8 & -60 & 60 \\
\hline DAN & MTG & 37 & -52 & -64 & 4 \\
\hline DAN & $\mathrm{PrCu}$ & 7 & 20 & -64 & 48 \\
\hline DAN & MTG & 37 & 48 & -60 & 4 \\
\hline DAN & SPL & 7 & 24 & -60 & 60 \\
\hline DAN & IPL & 40 & -32 & -48 & 48 \\
\hline DAN & $\mathrm{PrCu}$ & 19 & -28 & -72 & 36 \\
\hline DAN & MFG & 6 & -32 & 0 & 56 \\
\hline DAN & FFG & 37 & -44 & -60 & -8 \\
\hline DAN & SPL & 7 & -16 & -60 & 64 \\
\hline DAN & MFG & 6 & 28 & -4 & 52 \\
\hline FPN & PreCent & 6 & -44 & 0 & 44 \\
\hline FPN & MFG & 9 & 48 & 24 & 28 \\
\hline FPN & IFG & 9 & -48 & 12 & 24 \\
\hline FPN & IPL & 40 & -52 & -48 & 44 \\
\hline FPN & SFG & 6 & -24 & 12 & 64 \\
\hline FPN & ITG & 20 & 60 & -52 & -12 \\
\hline FPN & $\mathrm{ACC}$ & 10 & 24 & 44 & -16 \\
\hline FPN & MFG & 10 & 32 & 56 & -12 \\
\hline FPN & PreCent & 6 & 48 & 8 & 32 \\
\hline FPN & PreCent & 6 & -40 & 4 & 32 \\
\hline FPN & MFG & 46 & -44 & 40 & 20 \\
\hline FPN & MFG & 10 & 40 & 44 & 16 \\
\hline FPN & IPL & 40 & 48 & -44 & 44 \\
\hline FPN & SPL & 7 & -28 & -56 & 48 \\
\hline FPN & IPL & 40 & 44 & -52 & 48 \\
\hline FPN & MFG & 6 & 32 & 16 & 56 \\
\hline FPN & $\mathrm{PrCu}$ & 39 & 36 & -64 & 40 \\
\hline FPN & IPL & 40 & -44 & -56 & 44 \\
\hline FPN & MFG & 6 & 40 & 20 & 40 \\
\hline FPN & MFG & 10 & -36 & 56 & 4 \\
\hline FPN & IFG & 10 & -40 & 44 & -4 \\
\hline FPN & SPL & 7 & 32 & -52 & 44 \\
\hline FPN & MFG & 46 & 44 & 48 & -4 \\
\hline FPN & MFG & 9 & -44 & 24 & 28 \\
\hline FPN & $\mathrm{mFG}$ & 8 & -4 & 28 & 44 \\
\hline Salience & $\mathrm{PrCu}$ & 7 & 12 & -40 & 52 \\
\hline Salience & Supramarginal & 40 & 56 & -44 & 36 \\
\hline Salience & MFG & 6 & 44 & 0 & 48 \\
\hline
\end{tabular}




\begin{tabular}{|c|c|c|c|c|c|}
\hline Salience & MFG & 9 & 32 & 32 & 28 \\
\hline Salience & IFG & 45 & 48 & 24 & 8 \\
\hline Salience & Insula & 13 & -36 & 20 & 0 \\
\hline Salience & Insula & 13 & 36 & 20 & 4 \\
\hline Salience & IFG & 47 & 36 & 32 & -4 \\
\hline Salience & Claustrum & $*$ & 32 & 16 & -8 \\
\hline Salience & $\mathrm{CG}$ & 32 & -12 & 24 & 24 \\
\hline Salience & $\mathrm{mFG}$ & 32 & 0 & 16 & 44 \\
\hline Salience & SFG & 10 & -28 & 52 & 20 \\
\hline Salience & $\mathrm{CG}$ & 32 & 0 & 32 & 28 \\
\hline Salience & $\mathrm{CG}$ & 32 & 4 & 24 & 36 \\
\hline Salience & $\mathrm{CG}$ & 32 & 12 & 24 & 28 \\
\hline Salience & SFG & 10 & 32 & 56 & 16 \\
\hline Salience & SFG & 9 & 28 & 48 & 28 \\
\hline Salience & MFG & 10 & -40 & 52 & 16 \\
\hline SSM & $\mathrm{PrCu}$ & 7 & -8 & -52 & 60 \\
\hline SSM & $\mathrm{CG}$ & 24 & -12 & -16 & 40 \\
\hline SSM & ParaCent & 31 & 0 & -16 & 48 \\
\hline SSM & $\mathrm{CG}$ & 24 & 8 & 0 & 44 \\
\hline SSM & $\mathrm{mFG}$ & 6 & -8 & -20 & 64 \\
\hline SSM & ParaCent & 4 & -8 & -32 & 72 \\
\hline SSM & ParaCent & 4 & 12 & -32 & 76 \\
\hline SSM & PostCent & 2 & -52 & -24 & 44 \\
\hline SSM & PreCent & 4 & 28 & -16 & 72 \\
\hline SSM & PostCent & 7 & 8 & -44 & 72 \\
\hline SSM & PostCent & 2 & -24 & -32 & 72 \\
\hline SSM & PostCent & 3 & -40 & -20 & 56 \\
\hline SSM & Sub-Gyral & 40 & 28 & -40 & 60 \\
\hline SSM & PostCent & 2 & 52 & -20 & 40 \\
\hline SSM & PostCent & 2 & -40 & -28 & 68 \\
\hline SSM & ParaCent & 4 & 20 & -28 & 60 \\
\hline SSM & PreCent & 4 & 44 & -8 & 56 \\
\hline SSM & Sub-Gyral & 40 & -28 & -44 & 60 \\
\hline SSM & $\mathrm{mFG}$ & 6 & 12 & -16 & 76 \\
\hline SSM & SPL & 7 & 24 & -44 & 68 \\
\hline SSM & IPL & 40 & -44 & -32 & 48 \\
\hline SSM & Sub-Gyral & 40 & -20 & -32 & 60 \\
\hline SSM & PreCent & 6 & -12 & -16 & 76 \\
\hline SSM & PostCent & 3 & 44 & -20 & 56 \\
\hline SSM & PreCent & 4 & -40 & -16 & 68 \\
\hline SSM & PostCent & 7 & -16 & -44 & 72 \\
\hline SSM & ParaCent & 5 & 4 & -28 & 60 \\
\hline SSM & $\mathrm{mFG}$ & 6 & 4 & -16 & 60 \\
\hline SSM & PreCent & 4 & 36 & -16 & 44 \\
\hline
\end{tabular}




\begin{tabular}{|c|c|c|c|c|c|}
\hline SSM & IPL & 40 & 48 & -28 & 48 \\
\hline SSM & PreCent & 6 & -48 & -12 & 36 \\
\hline SSM & Claustrum & $*$ & 36 & -8 & 12 \\
\hline SSM & PreCent & 6 & 52 & -4 & 32 \\
\hline SSM & PreCent & 4 & 64 & -8 & 24 \\
\hline VAN & SFG & 6 & -8 & 12 & 68 \\
\hline VAN & STG & 13 & 52 & -44 & 20 \\
\hline VAN & STG & 22 & -56 & -52 & 8 \\
\hline VAN & STG & 22 & -56 & -40 & 12 \\
\hline VAN & STG & 41 & 52 & -32 & 8 \\
\hline VAN & STG & 22 & 52 & -28 & -4 \\
\hline VAN & STG & 22 & 56 & -48 & 12 \\
\hline VAN & IFG & 45 & 52 & 32 & 0 \\
\hline VAN & IFG & 45 & -48 & 24 & 0 \\
\hline Visual & Culmen & $*$ & 16 & -48 & -8 \\
\hline Visual & MOG & 19 & 40 & -72 & 16 \\
\hline Visual & $\mathrm{Cu}$ & 30 & 8 & -72 & 12 \\
\hline Visual & $\mathrm{LG}$ & 18 & -8 & -80 & 8 \\
\hline Visual & MOG & 19 & -28 & -80 & 20 \\
\hline Visual & LG & 19 & 20 & -64 & 0 \\
\hline Visual & MOG & 18 & -24 & -92 & 20 \\
\hline Visual & FFG & 19 & 28 & -60 & -8 \\
\hline Visual & LG & 18 & -16 & -72 & -8 \\
\hline Visual & LG & 19 & -16 & -68 & 4 \\
\hline Visual & FFG & 19 & 44 & -80 & -12 \\
\hline Visual & FFG & 19 & -48 & -76 & -8 \\
\hline Visual & $\mathrm{Cu}$ & 19 & -16 & -92 & 32 \\
\hline Visual & $\mathrm{Cu}$ & 19 & 16 & -88 & 36 \\
\hline Visual & $\mathrm{PrCu}$ & 31 & 28 & -76 & 24 \\
\hline Visual & LG & 18 & 20 & -84 & -4 \\
\hline Visual & $\mathrm{Cu}$ & 18 & 16 & -76 & 32 \\
\hline Visual & LG & 18 & -16 & -52 & 0 \\
\hline Visual & ITG & 19 & 40 & -64 & -8 \\
\hline Visual & $\mathrm{Cu}$ & 18 & 24 & -88 & 24 \\
\hline Visual & $\mathrm{Cu}$ & 18 & 4 & -72 & 24 \\
\hline Visual & ITG & 37 & -44 & -72 & 0 \\
\hline Visual & MOG & 19 & 24 & -80 & -16 \\
\hline Visual & $\mathrm{Cu}$ & 18 & -16 & -76 & 32 \\
\hline Visual & $\mathrm{Cu}$ & 18 & -4 & -80 & 20 \\
\hline Visual & IOG & 18 & -40 & -88 & -8 \\
\hline Visual & MOG & 19 & 36 & -84 & 12 \\
\hline Visual & LG & 18 & 8 & -80 & 8 \\
\hline Visual & MOG & 18 & -28 & -92 & 4 \\
\hline Visual & FFG & 19 & -32 & -80 & -12 \\
\hline
\end{tabular}


bioRxiv preprint doi: https://doi.org/10.1101/2021.07.27.453878; this version posted July 27, 2021. The copyright holder for this preprint (which was not certified by peer review) is the author/funder. All rights reserved. No reuse allowed without permission.
Visual
IOG
19
$\begin{array}{lll}36 & -80 & 0\end{array}$

Note: $\mathrm{CON}=$ Cingulo-opercular Task Control; DMN = Default Mode Network; DAN = Dorsal Attention Network; FPN = Fronto-parietal Task Control Network; SSM = Sensory/Somatomotor Network 
bioRxiv preprint doi: https://doi.org/10.1101/2021.07.27.453878; this version posted July 27, 2021. The copyright holder for this preprint (which was not certified by peer review) is the author/funder. All rights reserved. No reuse allowed without permission.

\section{Supplementary Figure 1. BPLS without regressing mean task-related activity}

LV1 (41.45\% cross-block covariance)

\section{A MEN}
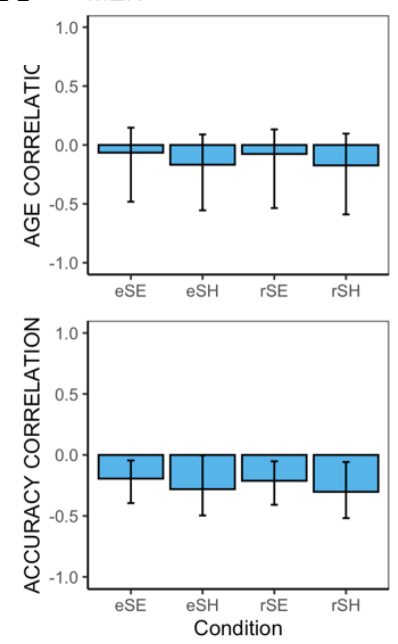

WOMEN
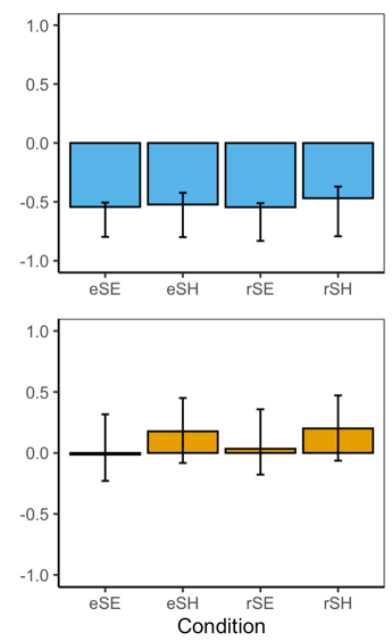

B
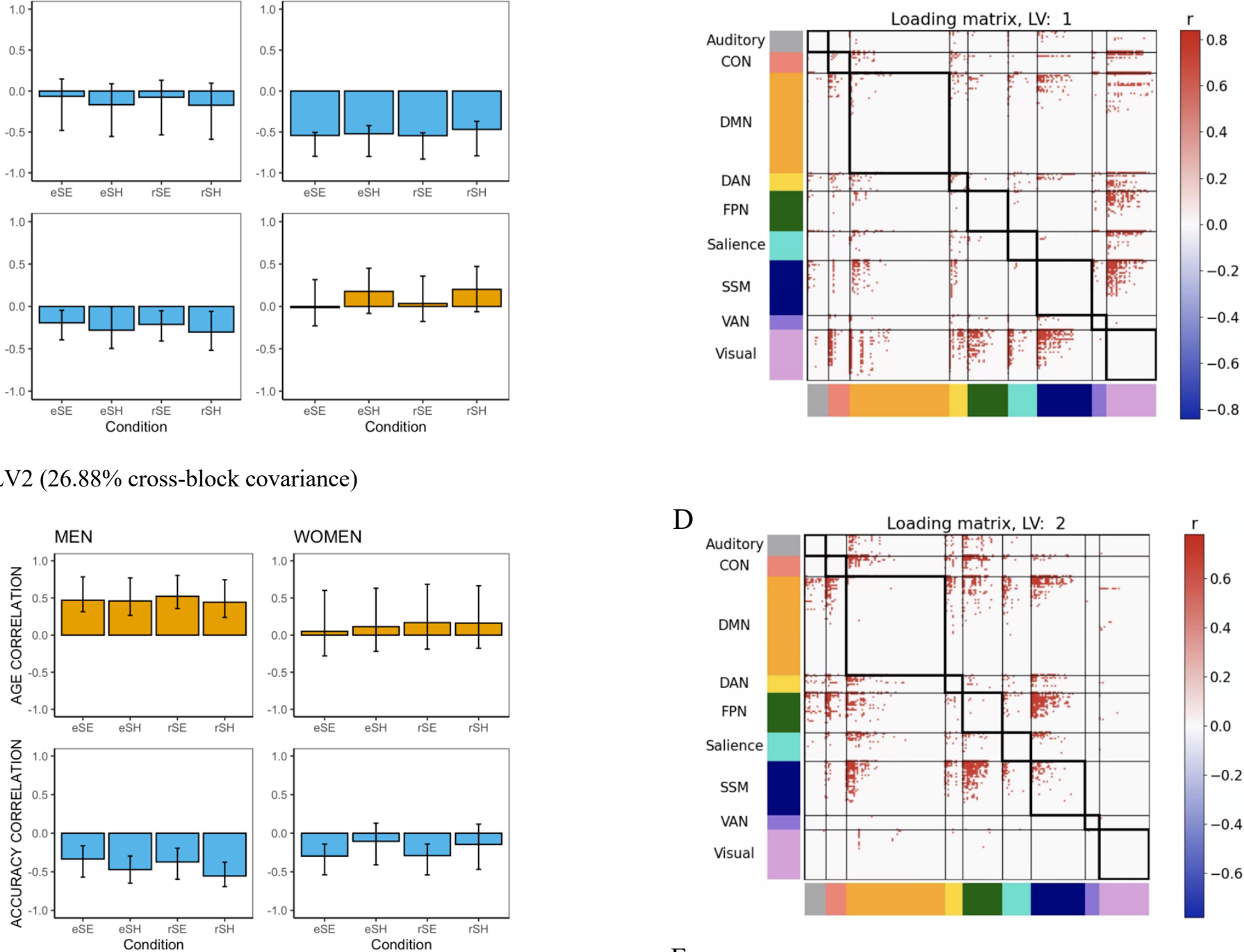

LV3 (9.37\% cross-block covariance)

$\mathrm{E}$
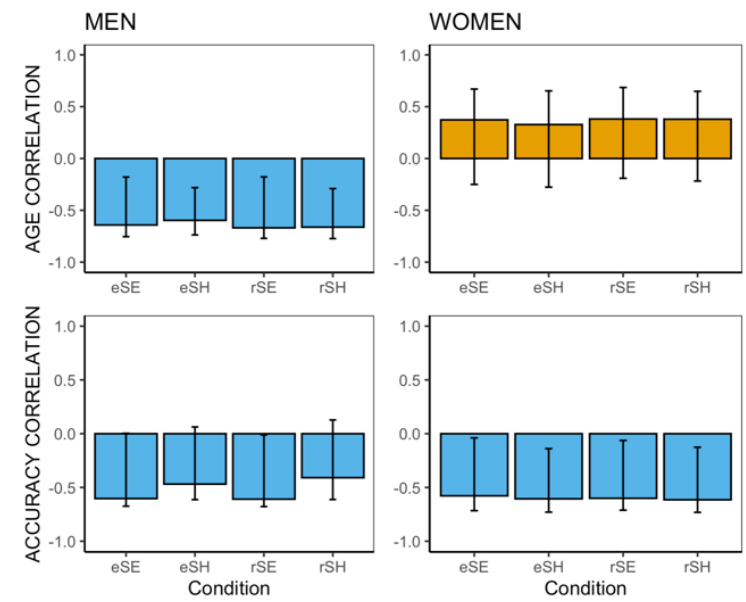

$\mathrm{D}$

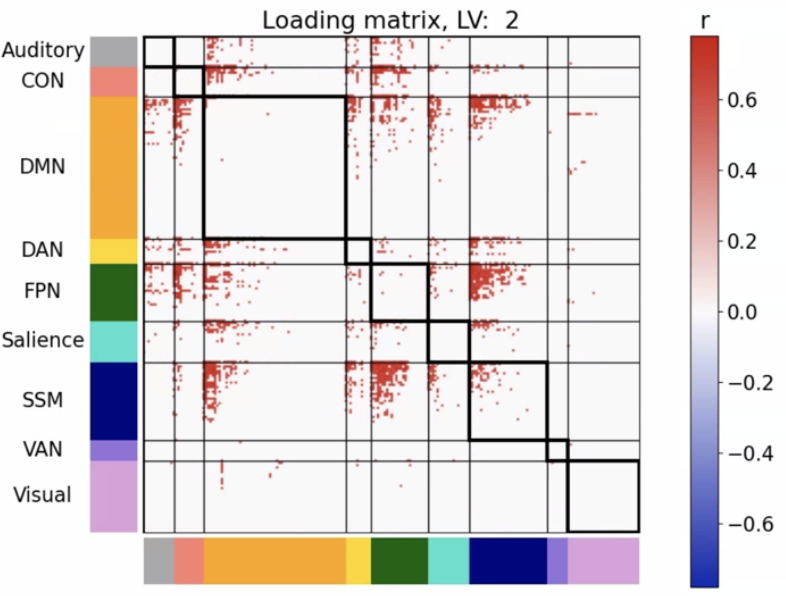

F

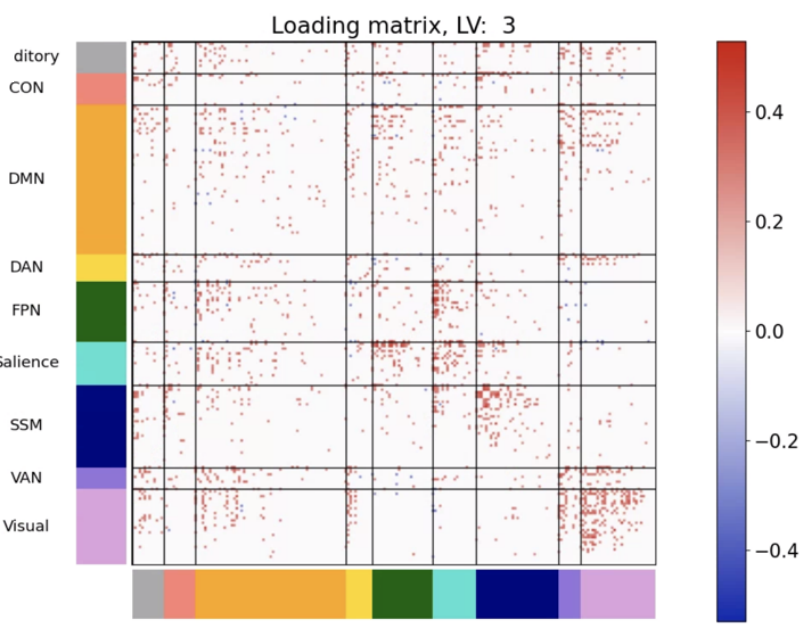

Note. The B-PLS analysis using connectivity matrices that did not regress mean-task-related activity generated 3 significant LVs, similar to the primary BPLS described in the manuscript. Supp Fig 1A, C, E reflect the correlation between the behavioural vectors of age and accuracy with the task fMRI connectivity of participants. Error bars represent bootstrapped standard deviations. Supp Fig 1B, D, F reflect the thresholded 95th percentile of correlations between participants' task fMRI data and behavioural profile. 
bioRxiv preprint doi: https://doi.org/10.1101/2021.07.27.453878; this version posted July 27, 2021. The copyright holder for this preprint (which was not certified by peer review) is the author/funder. All rights reserved. No reuse allowed without permission.

\section{Supplementary Figure 2. Matched Intracranial Volume Cohort Analysis}

\section{LV1 (44.17\% cross-block covariance)}

A

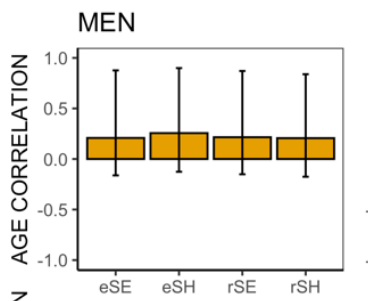

WOMEN
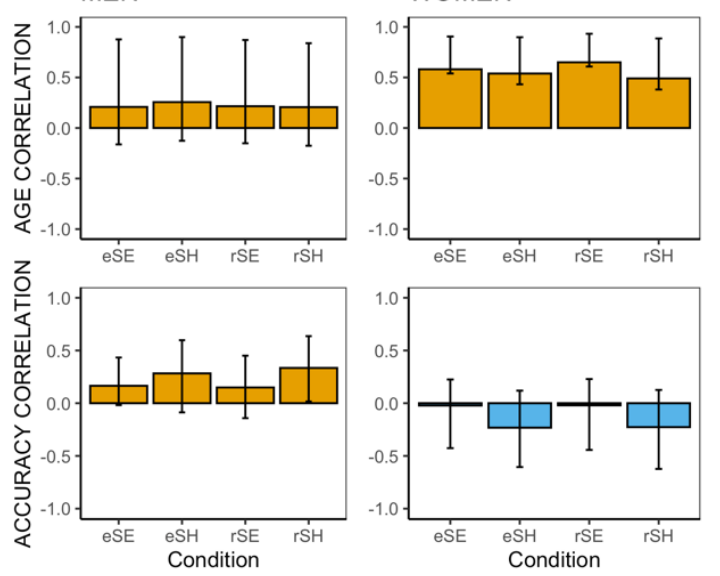

B

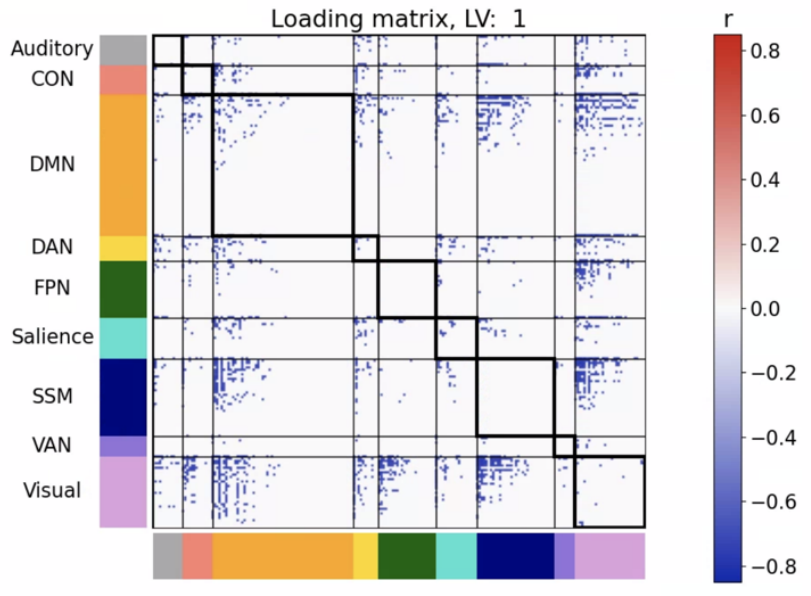

$\mathrm{D}$

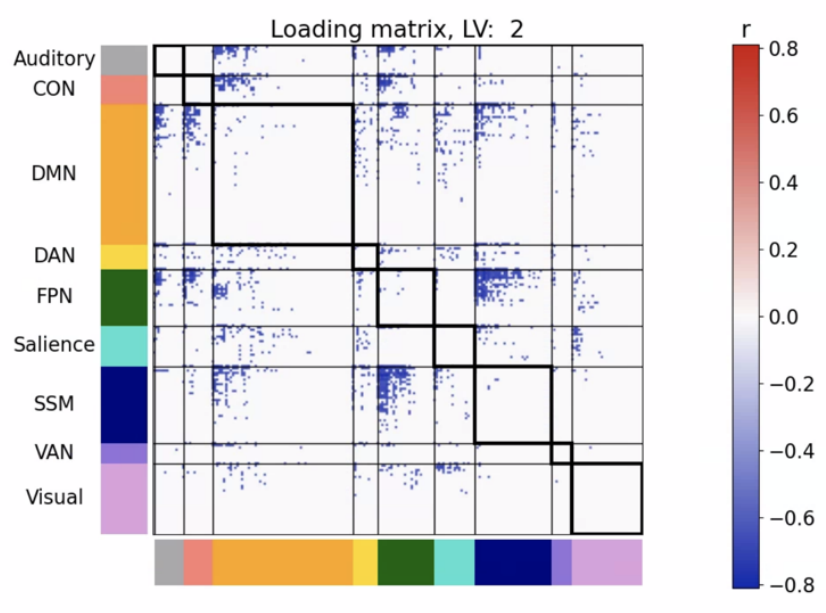

$\mathrm{F}$

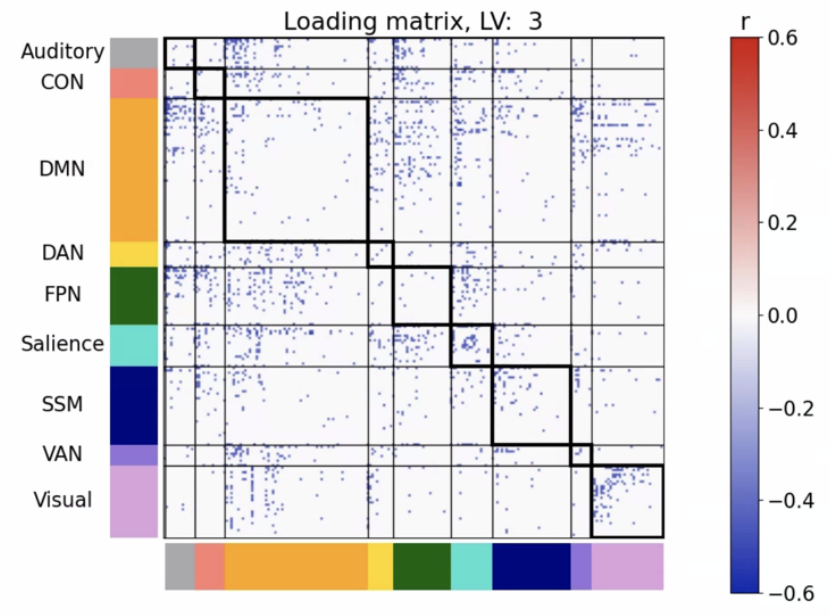

$\mathrm{E}$
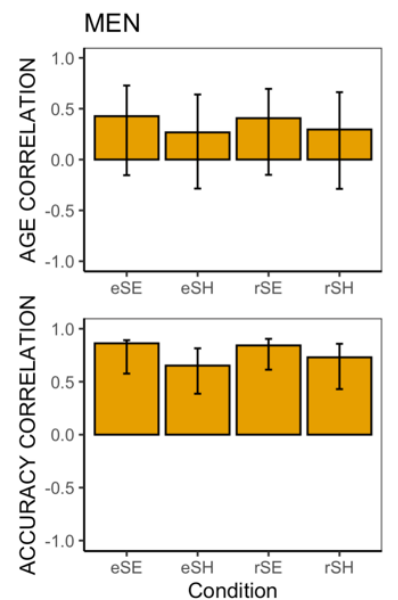

WOMEN
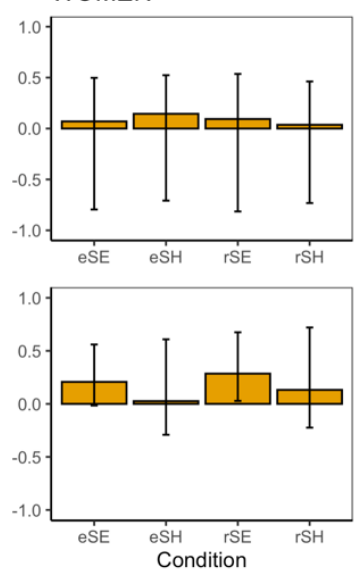

LV3 (9.92 \% cross-block covariance)

Note. The BPLS analysis with a subcohort of participants matched by intracranial volume, age, and education ( 25 men, 25 women) determined findings similar to the original BPLS analysis described in the manuscript. Supp Fig 1A, C, E reflect the correlation between the behavioural vectors of age and accuracy with the task fMRI connectivity of participants. Error bars represent bootstrapped standard deviations. Supp Fig 1B, D, F reflect the thresholded 95th percentile of correlations between participants' task fMRI data and behavioural profile 
bioRxiv preprint doi: https://doi.org/10.1101/2021.07.27.453878; this version posted July 27, 2021. The copyright holder for this preprint (which was not certified by peer review) is the author/funder. All rights reserved. No reuse allowed without permission.

Table 2. Mean Demographic and Behavioural Measures (and Standard Errors) in held-out Women $(\mathrm{n}=43)$

\begin{tabular}{lcccc} 
& Younger Adults (YA) & $\begin{array}{c}\text { Middle Aged Adults } \\
\text { (MA) }\end{array}$ & $\begin{array}{c}\text { Older Adults } \\
(\text { OA })\end{array}$ & $p$ \\
\hline Sample size (n) & 12 & 19 & 12 & \\
Age (years) & $25.75(1.45)$ & $51.74(1.08)$ & $64.25(0.76)$ & \\
Educations (years) & $14.75(0.77)$ & $15.53(0.52)$ & $14.67(0.62)$ & model ns \\
Predicted full-scale IQ & $118.84(1.72)$ & $118.32(1.13)$ & $119.22(2.06)$ & model ns \\
BDI & $3.33(1.10$ & $4.74(0.96)$ & $4.50(0.92)$ & model ns \\
CVLT-LFR & $14.92(0.29)$ & $13.68(0.34)$ & $13.33(0.72)$ & $\mathrm{p}<.05^{\#}$ \\
CVLT-LCR & $15.00(0.44)$ & $13.95(0.36)$ & $13.58(0.69)$ & model ns \\
CVLT-RG & $15.92(0.08)$ & $15.37(0.19)$ & $15.50(0.26)$ & model ns \\
BMI (kg/m ${ }^{2}$ ) & $22.10(1.40)$ & $25.43(1.03)$ & $26.83(1.23)^{*}$ & $\mathrm{p}<.01^{\#}$ \\
SE retrieval accuracy (\%correct) & $0.89(0.02)$ & $0.86(0.02)$ & $0.82(0.04)$ & model ns \\
SH retrieval accuracy (\%correct) & $0.90(0.03)$ & $0.82(0.03)$ & $0.78(0.04)$ & $\mathrm{p}<.01^{\#}$ \\
& & & 2856.97 & \\
SE retrieval RT (msec) & $2305.06(161.95)$ & $2477.96(112.40)$ & $(156.86)$ & $\mathrm{p}<.01^{\#}$ \\
SH retrieval RT (msec) & $2360.28(147.56)$ & $2622.64(92.27)$ & $(153.03)$ & $\mathrm{p}<.01^{\#}$ \\
\hline
\end{tabular}

*BMI missing for one participant $(\mathrm{n}=11)$. Values in brackets represent the standard error. A linear regression with Age as the independent measure was performed on each of the measures (significance of $p<.05$ used). \# The linear regression produced a significant main effect of Age. The fMRI behavioural measures revealed that OA participants performed significantly worse than younger and middle-aged participants and with significantly greater RT to complete the spatial tasks. BDI = Beck Depression Inventory; LFR = Long-form Free Recall; LCR = Long-form Cued Recall; RG = Recognition; BMI = body mass index.

Table 3. Mean Demographic and Behavioural Measures (and Standard Errors) in Women $(n=91)$

\begin{tabular}{lcccc} 
& Younger Adults (YA) & $\begin{array}{c}\text { Middle Aged Adults } \\
(\text { MA) }\end{array}$ & $\begin{array}{c}\text { Older Adults } \\
(\text { OA })\end{array}$ & $p$ \\
\hline Sample size (n) & 28 & 35 & 28 & \\
Age (years) & $25.96(0.74)$ & $49.69(0.90)$ & $66.14(0.65)$ & \\
Educations (years) & $15.89(0.44)$ & $15.66(0.36)$ & $15.07(0.42)$ & $\mathrm{ns}$ \\
Predicted full-scale IQ & $118.64(1.04)$ & $119.44(0.85)$ & $120.04(1.06)$ & $\mathrm{ns}$ \\
BDI & $3.44(3.95)^{*}$ & $4.54(0.72)$ & $3.79(0.57)$ & $\mathrm{ns}$ \\
CVLT-LFR & $14.11(0.29)$ & $13.46(0.27)$ & $13.25(0.44)$ & $\mathrm{ns}$ \\
CVLT-LCR & $14.25(0.34)$ & $13.71(0.30)$ & $13.39(0.39)$ & $\mathrm{ns}$ \\
CVLT-RG & $15.57(0.13)$ & $15.34(0.15)$ & $15.18(0.18)$ & $\mathrm{ns}$ \\
BMI (kg/m ${ }^{2}$ ) & $22.05(0.71)$ & $24.60(0.67)$ & $25.80(0.72)^{*}$ & $\mathrm{p}<.01^{\#}$ \\
SE retrieval accuracy (\%correct) & $0.89(0.01)$ & $0.86(0.02)$ & $0.83(0.02)$ & $\mathrm{p}<.01^{\#}$ \\
SH retrieval accuracy (\%correct) & $0.89(0.02)$ & $0.84(0.02)$ & $0.78(0.02)$ & $\mathrm{p}<.01^{\#}$ \\
SE retrieval RT (msec) & $2200.11(105.50)$ & $2464.40(9.95)$ & $2878.14(83.98)$ & $\mathrm{p}<.01^{\#}$ \\
SH retrieval RT (msec) & $2279.37(103.05)$ & $2610.75(77.30)$ & $2871.45(82.69)$ & $\mathrm{p}<.01^{\#}$ \\
\hline
\end{tabular}

*One participant had missing BDI information ( $\mathrm{n}=27)$, and one participant had missing BMI information ( $\mathrm{n}=27)$. Values in brackets represent the standard error. A linear regression with Age as the independent measure was performed on each of the measures (significance of $p<.05$ used). \# The linear regression produced a significant main effect of Age. The fMRI behavioural measures revealed that OA participants performed significantly worse than younger and middle-aged participants and with significantly greater RT to complete the spatial tasks. BDI = Beck Depression Inventory; LFR $=$ Longform Free Recall; LCR = Long-form Cued Recall; RG = Recognition; BMI = body mass index. 
bioRxiv preprint doi: https://doi.org/10.1101/2021.07.27.453878; this version posted July 27, 2021. The copyright holder for this preprint (which was not certified by peer review) is the author/funder. All rights reserved. No reuse allowed without permission.

\section{Supplementary Figure 3. Within-Sex PLS Analysis Within Women (n=91)}

A

LV1 $(71.39 \%$ cross-block covariance)
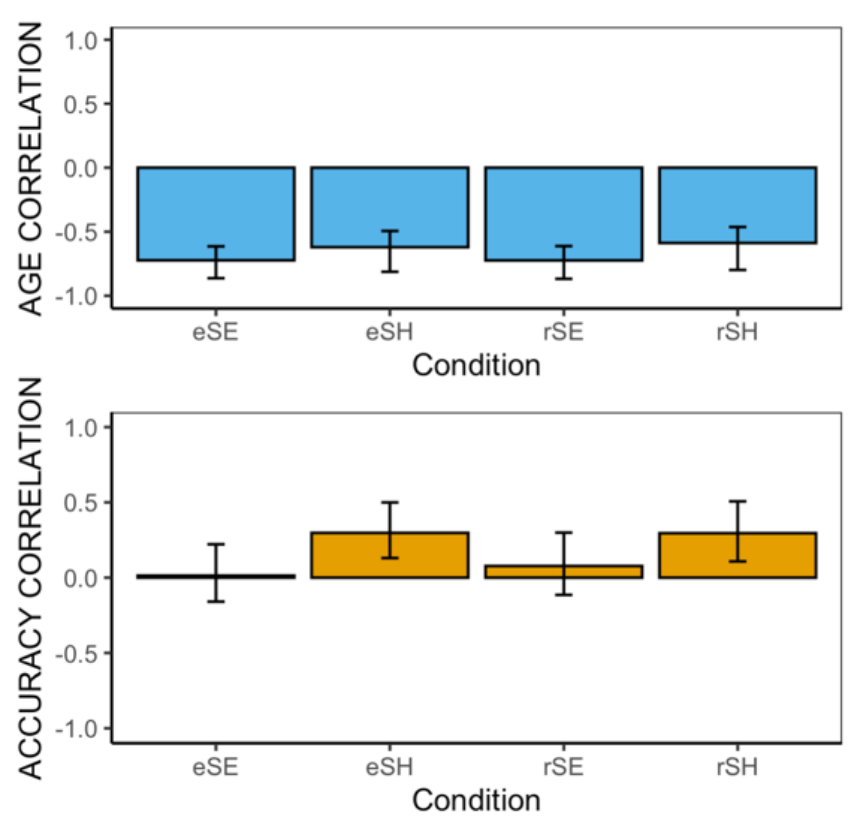

LV2 (13.96\% cross-block covariance)

$\mathrm{C}$
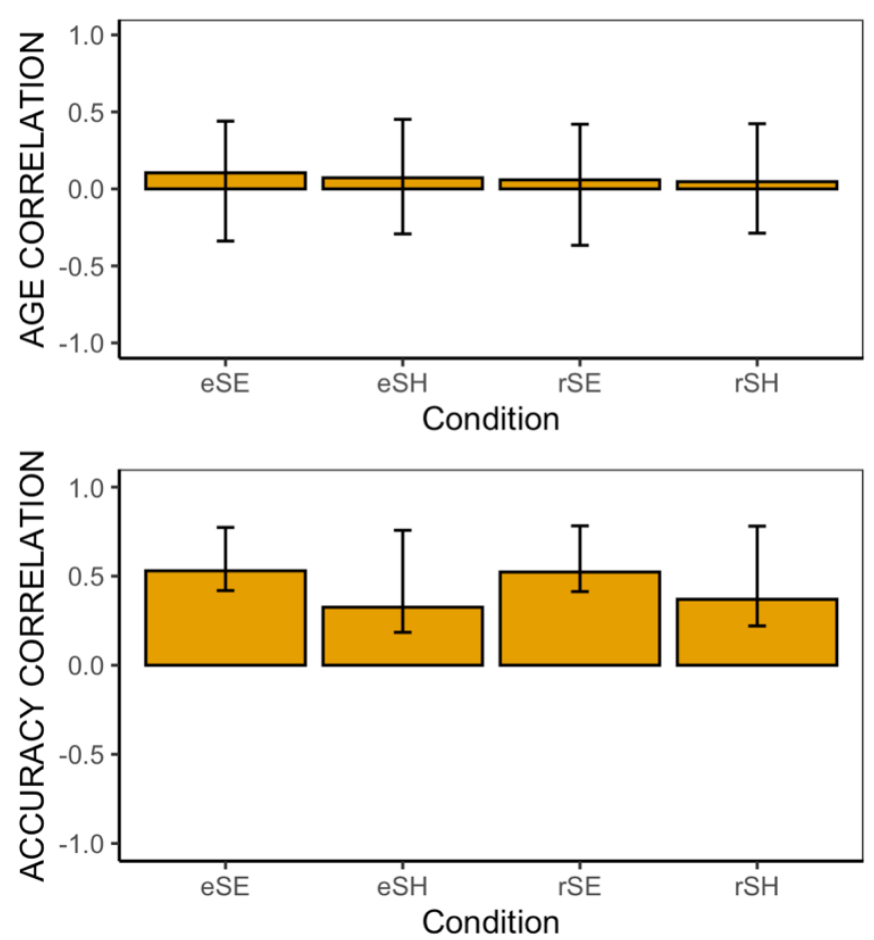

B

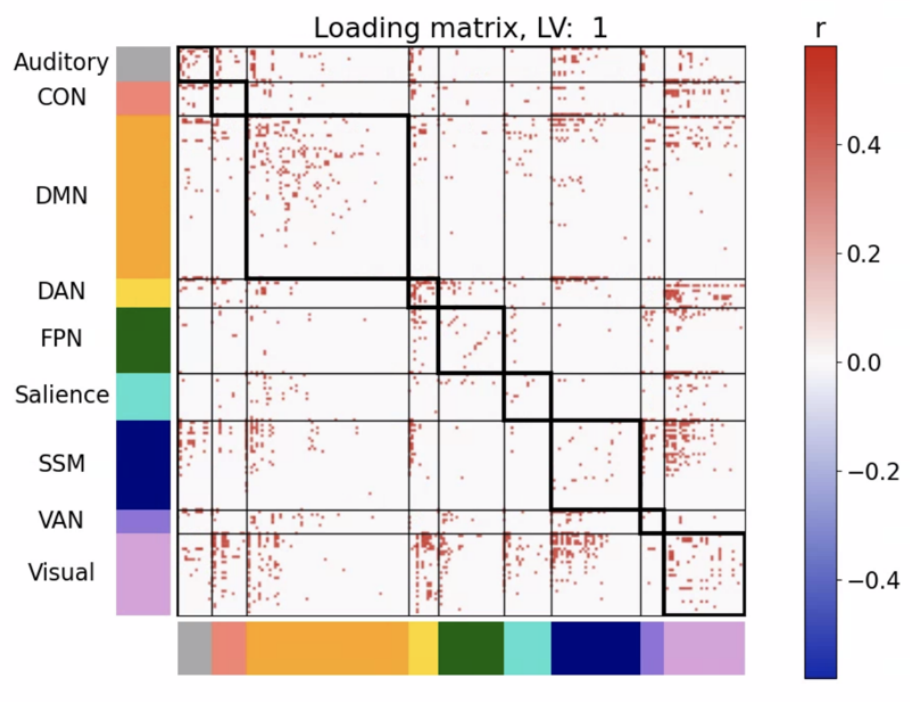

$\mathrm{D}$

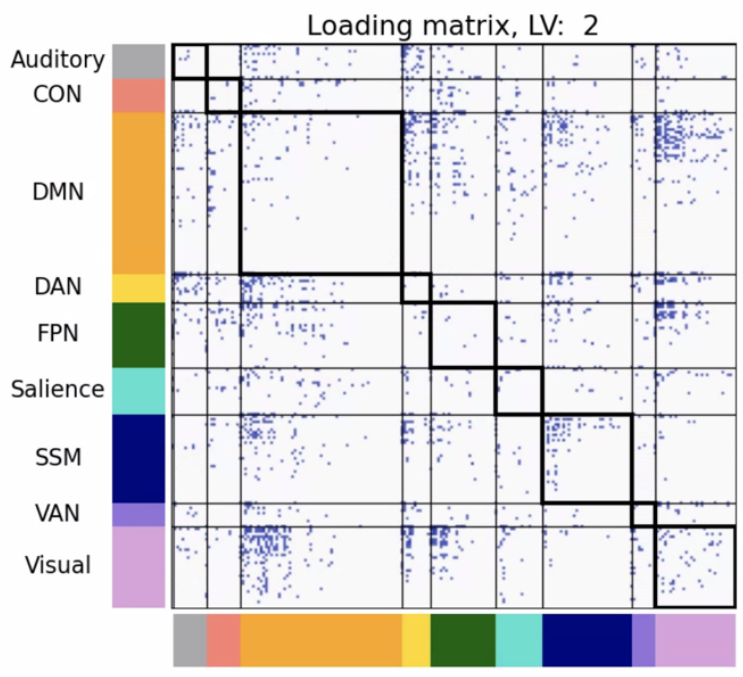

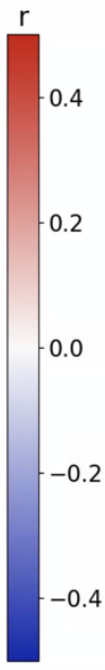

Note. The within-sex B-PLS of the full cohort of women $(\mathrm{n}=91)$ generated two significant LVs that produced findings consistent to the primary B-PLS findings in women. Supp Fig 1A and 1C reflect the correlation between the behavioural vectors of age and accuracy with the task fMRI connectivity of participants. Error bars represent bootstrapped standard deviations. Supp Fig 1B and 1D reflect the thresholded 95th percentile of correlations between participants' task fMRI data and behavioural profile. 